A) Check for updates

Cite this: Dalton Trans., 2018, 47 13616

\section{Synthesis of new allyl palladium complexes bearing purine-based NHC ligands with antiproliferative and proapoptotic activities on human ovarian cancer cell lines $\uparrow$}

\author{
Thomas Scattolin, ${ }^{a}$ Isabella Caligiuri, ${ }^{\mathrm{b}}$ Luciano Canovese, (D) ${ }^{\mathrm{a}}$ Nicola Demitri, ${ }^{\mathrm{c}}$ \\ Roberto Gambari, (D) d Ilaria Lampronti, (D) ${ }^{\mathrm{d}}$ Flavio Rizzolio, ${ }^{\text {abb }}$ Claudio Santo ${ }^{\mathrm{a}}$ and \\ Fabiano Visentin (1D *a
}

\begin{abstract}
A series of new palladium allyl complexes bearing purine-based carbenes derived from caffeine, theophylline and theobromine have been prepared and characterized by NMR spectroscopy, and elemental analysis and in two cases by single crystal X-ray diffraction. The cytotoxic and proapoptotic activities of compounds have been determined in vitro on human ovarian cancer A2780 and SKOV-3 cell lines. These experiments have shown that the palladium-allyl fragment induces a general cytotoxicity, but the choice of the supporting ligands is of paramount importance for achieving the best results. In particular complexes $\mathbf{4 c}, \mathbf{4 d}$ and $\mathbf{5 d}$ exhibit a higher antiproliferative effect $\left(\mathrm{IC}_{50}: 0.09,0.81\right.$ and $0.85 \mu \mathrm{M}$ respectively) than cisplatin $\left(\mathrm{IC}_{50}: 1.5 \mu \mathrm{M}\right)$ on $\mathrm{A} 2780$ cells, and $4 \mathrm{~d}\left(\mathrm{IC}_{50}: 1.7 \mu \mathrm{M}\right.$ vs. $\left.5.94 \mu \mathrm{M}\right)$ on SKOV-3 cell line. Moreover in many cases it has been proved that the cytotoxicity of our complexes is associated with the induction of apoptosis.
\end{abstract}

Received 7th May 2018,

Accepted 24th August 2018

DOI: $10.1039 / \mathrm{c} 8 \mathrm{dt} 01831 \mathrm{f}$

rsc.li/dalton target, thus altering its functionality. DNA is the most recurring genomic target and the ability of platinum to coordinate strongly to nucleic acid, forming intrastrand crosslinks, is the reason for the effectiveness of cisplatin and its second and third generation analogues. ${ }^{3,4}$ A lot of studies have subsequently proved that many potential metal-based anticancer drugs have non-genomic targets (usually proteins) regulating the cell cycle or inhibiting the over-expressed enzymes present in the cancer cells. ${ }^{2 d, 5}$

Unfortunately, a possible reactivity with biomolecules different from the specific diseased sites may induce a general toxicity, which is often the accompanying side effect of chemotherapeutic drugs.

In principle, a better selectivity could be obtained when the metal has a structural role i.e. it is helpful to achieve the appropriate shape of the complex. In these cases binding to the molecular target occurs through non-covalent interactions in a manner similar to most organic drugs. Within this context, the problem lies in the actual difficulty of precisely defining the shape and size of the target site and consequently synthesizing the appropriately tailored compounds. ${ }^{2 g}$

These preliminary remarks highlight that the rational design of a metal-based anticancer drug is an elegant but often impracticable strategy. Therefore an extensive screening of anti-tumour properties of different metal compounds may compensate for our inability to control their almost unpredict- 
able reactivity in the bloodstream and cellular environment. In our decision to contribute to this systematic search we have preliminarily set some guidelines suggested by previous contributions to the literature. Firstly, we have opted for palladiumbased compounds. Palladium complexes have structures and reactivity strictly comparable to platinum analogues and in several cases have shown better anti-cancer activity than cisplatin. ${ }^{6}$ However, the fast dissociation pattern of palladium complexes compared to platinum ${ }^{7}$ poses a problem since the speciation, which heavily affects the biological activity and the pharmacokinetic properties, might be increased. To remedy this contraindication the most direct option is to introduce ligands firmly anchored to the metal. For this reason we planned to employ $\mathrm{N}$-heterocyclic carbenes (NHCs), which are known to form strong $\sigma$-bonds with most of the transition metals. ${ }^{8}$ Moreover, several NHC-palladium complexes have already exhibited an interesting cytotoxic activity ${ }^{9,6 a, e}$ and tumour growth suppression even in vivo. ${ }^{6 e}$

A potential improvement introduced in our work is the use of some innovative NHC ligands with a purinic framework ${ }^{10}$ in the hope that the natural imprint of the moiety could make our palladium complexes more compatible with the biological matrix (Chart 1). This kind of synergy has already given interesting outcomes and in the most favourable cases the ligand has become a real targeting vector of the metal compound. ${ }^{11}$

Furthermore, with the aim of enhancing the versatility of our palladium complexes, sometimes also isocyanides or phosphines have been introduced into the coordination sphere. Among the latter we tested PTA (1,3,5-triaza-7-phosphadamantane) and TPPTS (3,3',3'--phosphinetribenzenesulfonate), which can increase the water-solubility of the metal compounds and thereby make their pharmacologic application easier. $^{12}$

However, the most original contribution of this paper is represented by the first attempt to utilize the palladium- $\eta^{3}$ allyl organometallic function for preparing compounds with cytotoxic properties. This organometallic fragment is well known for its involvement in numerous catalytic processes (the most famous is the Tsuji-Trost reaction) ${ }^{13}$ but its behaviour in biological systems remains an almost unexplored field. In previous studies, some of us have prepared numerous compounds based on this functional group and studied their properties and reactivity. ${ }^{14}$ This experience suggests that the allyl residue could: (a) remain bound to the metal centre, conferring a specific shape to the complex and thereby allowing it to be hosted in an active biological site; and (b) be released in the cellular environment and thus directly interact with some potential bio-target. The main goal of this paper is not to resolve this intricate question but analyse its practical consequences. Therefore, in order to verify the effects of our new palladium complexes, the potential antiproliferative activity was tested on two tumour cell lines (A2780 and SKOV-3) displaying a different sensitivity to cisplatin and for four representative complexes also on normal human fibroblasts MRC5. To obtain further information on the possible mechanism(s) of action, apoptosis was analysed as pro-apoptotic activity of palladium compounds. ${ }^{15}$

\section{Results and discussion}

\section{Synthesis of imidazolium salts from functionalized xanthines}

Compounds $\mathbf{1 b},{ }^{16 a} \mathbf{1 c}^{\mathbf{1 6 b}}$ and $\mathbf{1} \mathbf{d}^{17}$ were re-synthesized adopting a slightly modified literature procedure (Scheme 1). We have chosen conditions that are not particularly severe and the reaction of theophylline or theobromine with two equivalents of benzyl-bromide (for 1b and 1d) or 1-(phenyl)propargyl-3-chloride (for 1c), carried out in DMF at R.T. overnight, gave the functionalized bases in good yields (93-95\%).

Despite the low reactivity of the $\mathrm{sp}^{2} \mathrm{~N} 9$ atom, commercial caffeine (1a), the alkylated theophyllines $\mathbf{1 b}, \mathbf{1 c}$ and theobromine 1d react with a small excess of Meerwein's salt in $\mathrm{CH}_{3} \mathrm{CN}$ at R.T. in the presence of $\mathrm{Na}_{2} \mathrm{CO}_{3}$ under a non-controlled atmosphere to give the corresponding N9 methylated imidazolium salts in one hour's time (Scheme 2). Acetonitrile is probably more suitable for promoting the nucleophilic substitution than chlorinated solvents thanks to its high dielectric constant, whereas the presence of $\mathrm{Na}_{2} \mathrm{CO}_{3}$ in heterogeneous phase is necessary since the strong $\mathrm{HBF}_{4}$ acid that is partially formed
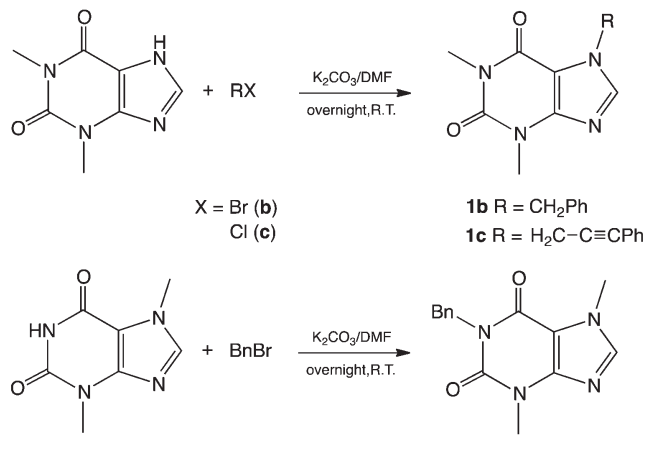

$1 d$

Scheme 1 Synthesis of functionalized xanthines.
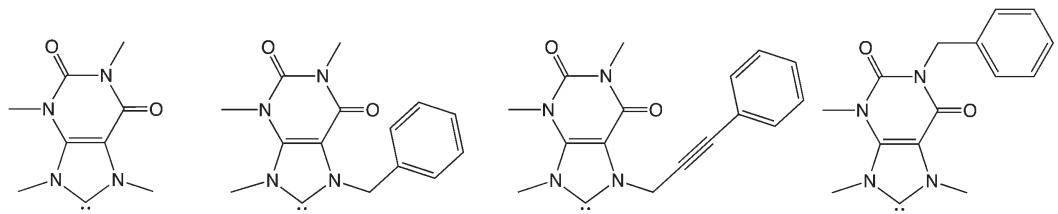

Chart 1 Purine-based NHC ligands used in this work. 

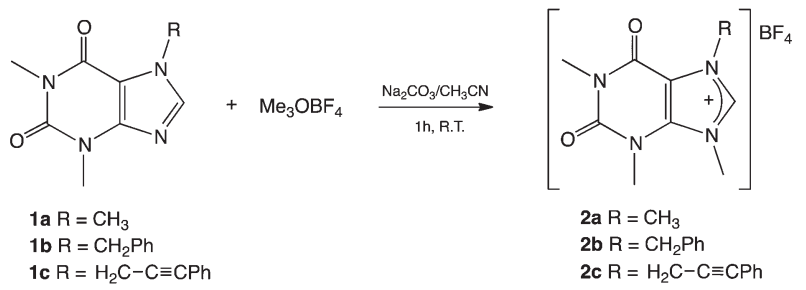

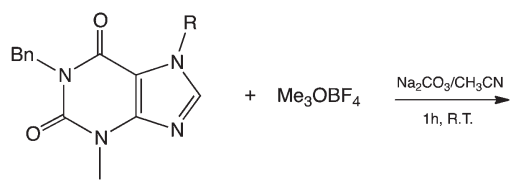

1d

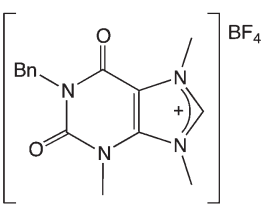

2d
Scheme 2 Synthesis of imidazolium salts from functionalized xanthines.

from the hygroscopic $\mathrm{Me}_{3} \mathrm{OBF}_{4}$ could protonate the N9 nitrogen of the xanthine so that the ensuing derivative would be no longer available for methylation.

As an example we report in Fig. S1 (see the ESI $\dagger$ ) the ${ }^{1} \mathrm{H}$ NMR spectra related to the methylation of caffeine (1a), which shows that, in the absence of $\mathrm{Na}_{2} \mathrm{CO}_{3}$, methyl caffeine $2 \mathrm{a}$ coexists with its protonated counterpart, whereas only pure 2 a derivative was detected in solution when the base was added to the reaction mixture.

In this respect, we think that the low yield obtained by other authors in Meerwein's salt mediated methylation of the modified xanthines is probably due to this side reaction, which makes the workup of the process difficult. ${ }^{15,18}$

\section{Synthesis of silver NHC complexes}

We recently reported that the reaction between the imidazolium salts $2 \mathbf{a}, \mathbf{2 b}, \mathbf{2 d}$ and $\mathrm{Ag}_{2} \mathrm{O}$ carried out in acetonitrile produces a 1:1 mixture of the corresponding silver bis-NHC complexes 3 and $\mathrm{AgBF}_{4} \cdot{ }^{19}$ This result was confirmed by a combination of spectroscopic and analytical data such as microanalysis, NMR, ESI-MS spectra and in one case (3b) even X-ray diffraction analysis. In this paper we have applied this protocol for the synthesis of the new complex 3c. The disappearance of the imidazolic proton and the typical shift of the silvercoordinated carbon at ca. $187 \mathrm{ppm}$ in the ${ }^{1} \mathrm{H}$ and ${ }^{13} \mathrm{C} \mathrm{NMR}$ spectra, respectively, represent simple tests to confirm the predictable outcome of the reaction (Scheme 3).

\section{Synthesis of mixed NHC/phosphine and NHC/isocyanide palladium $\eta^{3}$-allyl complexes}

For the synthesis of the palladium complexes 4-7 we can take advantage of the presence of co-precipitated $\mathrm{AgBF}_{4}$ as a stoichiometric by-product in the preparation of silver complexes 3 . As a matter of fact, this condition allows the final complexes to be obtained through a one-pot reaction, by mixing the aforesaid mixture with a stoichiometric amount of palladium allyl dimer and phosphine $\left(\mathrm{PPh}_{3}, \mathrm{PTA}\right.$, TPPTS $)$ or isocyanide (DIC $=$ 2,6-dimethylphenyl isocyanide) (Scheme 4). The precipitation of silver chloride is the driving force of the process and an indication of its progress. It is important to highlight that no formation of complexes bearing carbene/carbene and phosphine-phosphine (or isocyanide-isocyanide) ligands has been observed and therefore a selective separation of the carbene/ phosphine (or isocyanide) complexes is always possible from the reaction mixture.

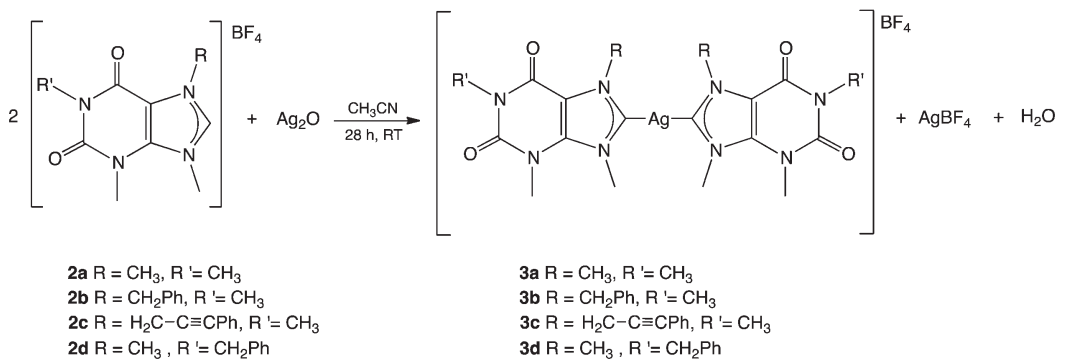

Scheme 3 Synthesis of silver NHC purine-based complexes.

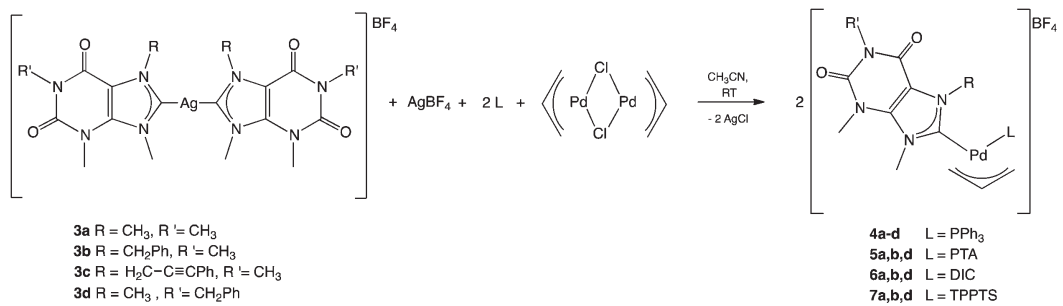

Scheme 4 Synthesis of mixed NHC/phosphine and NHC/isocyanide palladium allyl complexes. 
The characterization of the thirteen new complexes $4 a-d$,

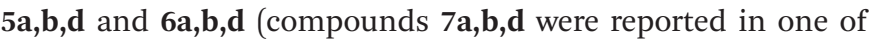
our previous studies ${ }^{19}$ ) was performed by NMR, IR and elemental analysis. In particular, an accurate examination of NMR spectra allows some specific features of these derivatives to be elucidated, which we can summarize on the following points:

(a) In every NMR spectrum of the complexes containing a coordinated phosphine $(\mathbf{4 a}-\mathbf{d}$ and $\mathbf{5 a}, \mathbf{b}, \mathbf{d})$ the presence of two complete sets of signals is manifest. This is a consequence of the coexistence of a couple of atropoisomers due to the hindered rotation of the asymmetric carbene ligands around the $\mathrm{Pd}-\mathrm{C}$ bond. The two isomers are always present in virtually equal amounts.

For the less sterically crowded isocyanide-complexes $\mathbf{6 a , b}, \mathbf{d}$ this restriction is not operative and thus only one set of signals is observable in solution at room temperature.

(b) The presence in the ${ }^{1} \mathrm{H}$ NMR spectra of five distinct signals (for each atropoisomer) ascribable to allyl protons can be explained by the presence of two different spectator ligands and by the absence of a rearrangement $\left(\eta^{3}-\eta^{1}-\eta^{3}\right.$ or $s y n-s y n /$ anti-anti) sometimes observed even at room temperature. ${ }^{20}$

(c) The coordination of $\mathrm{PPh}_{3}$ and PTA (for complexes $4 \mathbf{4}-\mathbf{d}$ and $\mathbf{5 a}, \mathbf{b}, \mathbf{d}$, respectively) is proved by the marked downfield shift of the two peaks (one for each atropoisomer) observed in the ${ }^{31} \mathrm{P}\left\{{ }^{1} \mathrm{H}\right\}$ NMR spectra $(\Delta \delta=30-45 \mathrm{ppm}$ with respect to the free phosphines).

(d) The coordination of DIC in complexes $\mathbf{6 a , b , \mathbf { d }}$ is confirmed by the weak signal of the coordinated isocyanide carbon at $\sim 150 \mathrm{ppm}$ in the ${ }^{13} \mathrm{C}\left\{{ }^{1} \mathrm{H}\right\}{ }^{1} \mathrm{H}$ NMR spectra and the significantly highfield shift of the ortho methyl protons with respect to their original position in the ${ }^{1} \mathrm{H}$ NMR spectra of the free ligand. This conclusion is also supported by the IR spectra, which show an intense band at $2170 \mathrm{~cm}^{-1}$, attributable to the $\mathrm{CN}$ stretching of the coordinated isocyanide.

(e) The resonances of carbene carbons are always found in a narrow range between 180 and 190 ppm, confirming the coordination of the purine-based NHC ligand at the palladium (II) centre. Moreover, in the spectra of compounds $4 \mathbf{4}-\mathbf{d}$ and $\mathbf{5 a}, \mathbf{b}, \mathbf{d}$, these signals resonate as doublets due to the $J^{2}$ coupling with the cis-phosphine.

The cationic nature of synthesized derivatives is indirectly confirmed by the presence in all IR spectra of an intense absorption at around $1050 \mathrm{~cm}^{-1}$, ascribable to the $\mathrm{BF}_{4}{ }^{-}$anion.

\section{Synthesis of bis(NHC) palladium $\eta^{3}$-allyl complexes}

This class of complexes were synthesized by reacting a stoichiometric mixture of silver precursors 3 and $\mathrm{AgBF}_{4}$ with palladium allyl dimer in the presence of KI (Scheme 5). The addition of potassium iodide is necessary to remove all the silver from the reaction mixture.

Transmetallation proceeds in good yield and the resulting cationic complexes were all stable in solution and in air.

The most significant conclusion that can be immediately inferred from the NMR spectra of species $\mathbf{8}$ is the presence of only one set of signals. This observation indicates that there is a free rotation of the two N-heterocyclic carbene ligands around the $\mathrm{Pd}-\mathrm{C}$ bond and thus the three hypothetical atropoisomers deriving from hindered rotation are not distinguishable at room temperature.

The symmetric structure of these complexes is demonstrated by the presence of only three signals ascribable to the allylic fragment in the ${ }^{1} \mathrm{H}$ NMR spectra (two doublets with different coupling constants for the syn and anti terminal protons and a multiplet for the central proton) and two in the ${ }^{13} \mathrm{C}\left\{{ }^{1} \mathrm{H}\right\} \mathrm{NMR}$ spectra.

Finally, the ${ }^{13} \mathrm{C}_{\mathrm{NHC}}-\mathrm{Pd}(\mathrm{II})$ resonances at about $185 \mathrm{ppm}$ represent compelling evidence of the coordination of carbene ligands to the palladium centre.

\section{X-Ray crystal structure determination of $4 \mathrm{a}$ and $8 \mathrm{~d}$}

Crystalline forms of $\mathbf{4 a}$ and $\mathbf{8 d}$ contain one crystallographically independent palladium complex (Fig. 1 and 2). The complexes

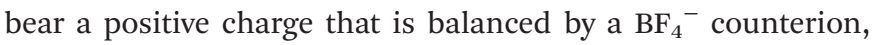
located close to the allyl ligand, which represents the area where the metal is more exposed (the shortest F...Pd contacts are 3.50(1) $\AA$ in $\mathbf{4 a}$ and 3.489(8) $\AA$ in 8d). Palladium adopts square planar coordination spheres with bond lengths and angles (Table $2 \mathrm{SI} \dagger$ ) in agreement with the literature structural data of complexes with similar ligands. A query on CSD (version 5.38) using the allyl-Pd-NHC fragment produces 29 hits with an average bite angle of $68.3(6)^{\circ}$ and 2.04(1) $\AA$ Pd $\cdots \mathrm{C}_{\text {carbenic }}$ bond lengths (2.32(1) $\AA$ for Pd...P bonds). The allyl ligand has poor steric bulk constraints and can adopt alternative conformations, specular with respect to the palladium coordination plane, as can be seen in more than half of similar structures already published. Models show that the xanthines minimize steric repulsions in the solid state, adopt-

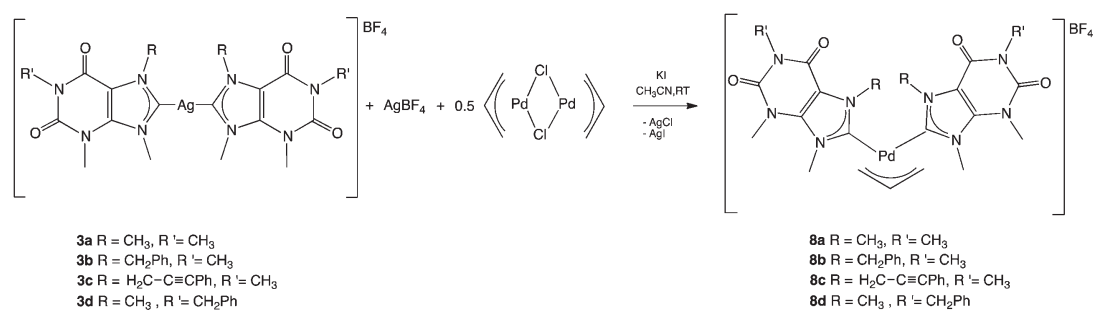

Scheme 5 Synthesis of bis(NHC) palladium allyl complexes. 


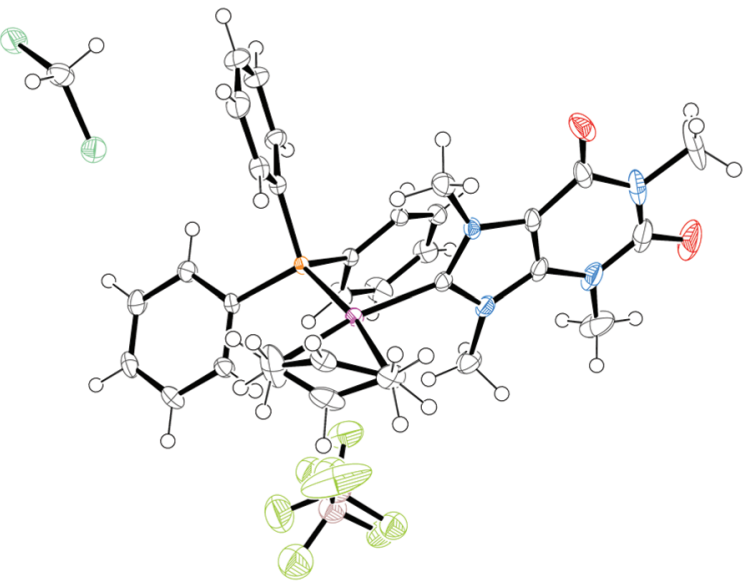

Fig. 1 Ellipsoid representation of 4 a (B) crystal ASU contents $(50 \%$ probability).

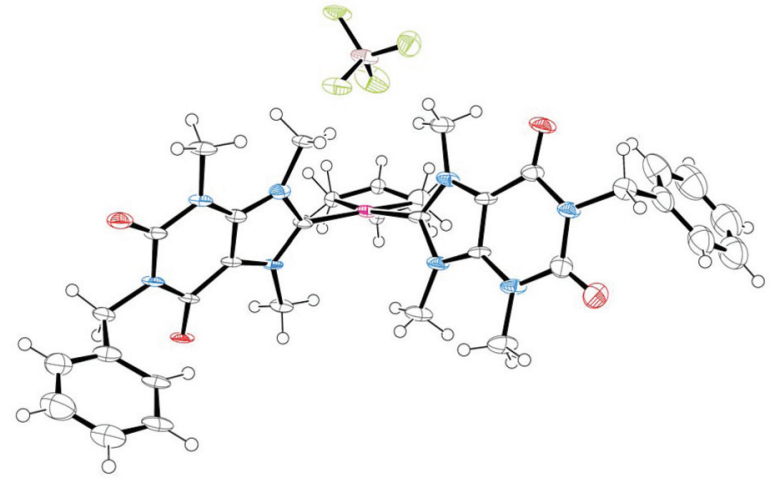

Fig. 2 Ellipsoid representation of 8d (B) crystal ASU contents $(50 \%$ probability).

ing a roughly perpendicular orientation with respect to the palladium coordination plane $\left(85.68^{\circ}\right.$ in $\mathbf{4 a}$ and $69.19^{\circ}-75.98^{\circ}$ in 8d, in agreement with the average $79(9)^{\circ}$ extracted from CSD). The molecular model of $\mathbf{4 a}$ is well superimposable with the related triphenylphosphine-( $\eta^{3}$-allyl)-(tetramethylimidazolin2-ylidene)-palladium tetrafluoroborate complex ${ }^{21}$ (CCDC number: 714135); the comparison highlights a degree of phosphine ligand conformational freedom (Fig. S27†), which can be related to different crystal packing contacts. Crystal packing of $\mathbf{4 a}$ and $\mathbf{8 d}$ shows hydrophobic contacts among neighbour molecules, involving several $\mathrm{CH} \cdots \pi$ and minor $\pi \cdots \pi$ interactions. Furthermore, structure $4 \mathbf{a}$ has cavities $\left(258 \AA^{3}\right.$, estimated using the PLATON ${ }^{22}$ 'CALC VOID' routine) filled with disordered solvent molecules (one $\mathrm{CH}_{2} \mathrm{Cl}_{2}$ molecule for each cavity).

\section{Antiproliferative activity on human A2780, SKOV-3 and MRC-5 cell lines}

The antiproliferative activity was assayed on two human cancer cell lines: A2780 and SKOV-3. Stock solutions (25-50 mM) of each complex were prepared in DMSO (8), $\mathrm{H}_{2} \mathrm{O}$ (5 and 7), or
DMSO/ $\mathrm{H}_{2} \mathrm{O}(1 / 1)$ (4 and 6); the working solutions (5 $\mathrm{mM}$, $500 \mu \mathrm{M}, 50 \mu \mathrm{M}$ and $5 \mu \mathrm{M})$ were obtained using $\mathrm{H}_{2} \mathrm{O}$ only. Preliminarily the stability of complexes was checked in a 1:1 DMSO-d6/ $\mathrm{D}_{2} \mathrm{O}$ solution (4, 6 and 8) or $\mathrm{D}_{2} \mathrm{O}(5$ and 7). After 48 hours at room temperature no degradation and no ligand replacement was observed (see NMR spectra in Fig. S28-S30†).

All of the complexes $\mathbf{4 a - d , 5 a , b , d , ~ 6 a , b , d , ~ 7 a , b , d , ~ a n d ~} \mathbf{8 a - d}$ and the synthetic precursor $\left[\mathrm{Pd}(\mu-\mathrm{Cl})\left(\eta^{3}-\mathrm{C}_{3} \mathrm{H}_{5}\right)\right]_{2}$ have been tested on the cisplatin-sensitive A2780 cell line and on the cisplatin-resistant SKOV-3 cell line in comparison with cisplatin (positive control) in order to evaluate the relative $\mathrm{IC}_{50}$ values expressed in $\mu \mathrm{M}$ concentration. All the data obtained are reported in Table 1 for both the analysed cell lines. As expected, cisplatin was more active on $\mathrm{A} 2780$ cells $\left(\mathrm{IC}_{50}=1.5 \pm\right.$ $0.2 \mu \mathrm{M})$ with respect to SKOV-3 cells $\left(\mathrm{IC}_{50}=5.94 \pm 0.08 \mu \mathrm{M}\right)$. In addition the results reported in Table 1 show that all the complexes, except 7a, displayed good anti-proliferative effects on the cisplatin-sensitive A2780 cells. The most active of them are derivatives $\mathbf{4 c}, \mathbf{4 d}$ and $\mathbf{5 d}$, which exhibit a greater activity than the positive control cisplatin $\left(\mathrm{IC}_{50}: 1.5 \pm 0.2 \mu \mathrm{M}\right.$ ), showing $0.09 \pm 0.02,0.81 \pm 0.08$ and $0.8 \pm 0.2 \mu \mathrm{M} \mathrm{IC}_{50}$ values, respectively. On the cisplatin-resistant SKOV-3 cells, several compounds (5d, 7a, 7b, 7d, 8b, 8c and 8d) exhibited lower activity $\left(\mathrm{IC}_{50}>30 \mu \mathrm{M}\right)$ with respect to cisplatin whereas most of them displayed a similar activity $\left(\mathrm{IC}_{50}=3-8 \mu \mathrm{M}\right)$. Remarkably, compounds $\mathbf{4 b}, \mathbf{5 a}, \mathbf{5} \mathbf{b}, \mathbf{6} \mathbf{a}$, and $\mathbf{6} \mathbf{d}$ exhibited a higher activity on SKOV-3 cells with respect to A2780 cells. Furthermore, complex 4d showed a very interesting activity on SKOV-3, exhibiting an $\mathrm{IC}_{50}$ value $(1.7 \pm 0.9 \mu \mathrm{M})$ lower than that of cisplatin. The imidazolium salts $\mathbf{2 a}$ and $\mathbf{2 b}$, also tested on the same cell models, were completely inactive (data not shown) at the concentrations used on both cell lines $\left(\mathrm{IC}_{50}>100 \mu \mathrm{M}\right)$.

To test the selectivity of Pd-complexes versus cancer cells, normal human fibroblasts (MRC-5) were treated with four

Table 1 Effects of the Pd-complexes on the proliferation of A2780 and SKOV-3 cells. The inhibition of cell growth is denoted as $I C_{50}$

\begin{tabular}{|c|c|c|}
\hline \multirow[b]{2}{*}{ Complex } & \multicolumn{2}{|l|}{$\mathrm{IC}_{50}(\mu \mathrm{M})$} \\
\hline & $\mathrm{A} 2780\left(\mathrm{IC}_{50}\right)$ & SKOV-3 $\left(\mathrm{IC}_{50}\right)$ \\
\hline Cisplatin & $1.5 \pm 0.2$ & $5.94 \pm 0.08$ \\
\hline$\left[\mathrm{Pd}(\mu-\mathrm{Cl})\left(\eta^{3}-\mathrm{C}_{3} \mathrm{H}_{5}\right)\right]_{2}$ & $7.8 \pm 0.2$ & $10 \pm 4$ \\
\hline $4 a$ & $4 \pm 2$ & $5 . \pm 1$ \\
\hline $4 b$ & $4 \pm 2$ & $3 \pm 2$ \\
\hline $4 c$ & $0.09 \pm 0.02$ & $4.02 \pm 0.09$ \\
\hline $4 d$ & $0.81 \pm 0.08$ & $1.7 \pm 0.9$ \\
\hline $5 \mathbf{a}$ & $7 \pm 2$ & $5.20 \pm 0.08$ \\
\hline $5 \mathbf{b}$ & $7.60 \pm 0.07$ & $6.5 \pm 0.7$ \\
\hline $5 d$ & $0.8 \pm 0.2$ & $50.5 \pm 0.5$ \\
\hline $6 a$ & $5 \pm 2$ & $4.0 \pm 0.7$ \\
\hline $6 b$ & $3.72 \pm 0.06$ & $5.2 \pm 0.9$ \\
\hline $6 d$ & $5 \pm 1$ & $3 \pm 1$ \\
\hline $7 \mathbf{a}$ & $80 \pm 10$ & $70 \pm 10$ \\
\hline $7 \mathbf{b}$ & $7 \pm 2$ & $40 \pm 2$ \\
\hline $7 d$ & $9.2 \pm 0.8$ & $57 \pm 8$ \\
\hline $8 \mathbf{a}$ & $7 \pm 2$ & $7.7 \pm 0.4$ \\
\hline $8 b$ & $4 \pm 1$ & $61 \pm 7$ \\
\hline $8 c$ & $3.7 \pm 0.5$ & $60 \pm 6$ \\
\hline $8 d$ & $6.3 \pm 0.7$ & $38 \pm 6$ \\
\hline
\end{tabular}


different compounds (4d, $\mathbf{5 d}, \mathbf{6 b}, \mathbf{8 a})$ representative of different subclasses with high activity on cancer cells. As shown in Table $\mathrm{S} 1, \uparrow$ the compounds are almost inactive on fibroblasts, suggesting a preferential activity on cancer cells. In particular, the compound $\mathbf{4 d}$ is more active than $\mathbf{5 d}, \mathbf{6 b}$, and $\mathbf{8 a}$ on A2780 and SKOV3 cancer cell lines but with an $\mathrm{IC}_{50}>100 \mu \mathrm{M}$ on fibroblasts.

\section{Pro-apoptotic effects on human A2780 and SKOV-3 cell lines}

In order to examine whether the antiproliferative activity of the analysed Pd-complexes is associated with induction of apoptosis, both A2780 and SKOV-3 cells were also evaluated employing the Annexin $\mathrm{V}$ release assay and a Muse cytometer (Merck Millipore, Billerica, MA, USA), after treatment with two concentrations close to the $\mathrm{IC}_{50}$ values previously determined. The found pro-apoptotic effects are reported in Table 2, Fig. 3 and 4 (representative examples) and in Fig. $1 \mathrm{~A}-\mathrm{E}$ and $2 \mathrm{~A}-\mathrm{E}$ in the ESI† (all results). In Table 2, the percentage of total apoptosis is reported. Each data entry is compared to the analysis of untreated cells (negative control, $\mathbf{C}-$ ) in which the observed total apoptosis was $\leq 5 \%$.

On the A2780 cell line, the complexes that showed the highest pro-apoptotic activity were $4 \mathbf{c}, 4 \mathbf{d}, \mathbf{6 b}, \mathbf{6 d}, \mathbf{8 b}, \mathbf{8 c}$, and 8d, with total pro-apoptotic effects between $21.00 \%$ (4d) and 98.35\% (4c); in addition, complex 4c showed the greatest total pro-apoptotic activity (54.04\%) even with the lowest concentration tested $(0.1 \mu \mathrm{M})$. The remaining compounds were found to be moderately or poorly active in inducing apoptosis on this cisplatin-sensitive cell line, and the synthetic precursor $\left[\mathrm{Pd}(\mu-\mathrm{Cl})\left(\eta^{3}-\mathrm{C}_{3} \mathrm{H}_{5}\right)\right]_{2}$ had a pro-apoptotic effect on $29.40 \%$ of cells.

On the cisplatin-resistant SKOV-3 cell line, derivatives $\mathbf{4 b}$, 4c, 4d, 5d, 6a, 6b, 6d, 7a, 7b, 7d, 8a, 8b, 8c, and 8d were found to be particularly active, with total pro-apoptotic effects between $27.49 \%$ (5d) and $84.04 \%$ (4c). In particular, complex 4c showed the highest activity even at the lowest concentration used $(1 \mu \mathrm{M})$ with $43.78 \%$ of apoptotic cells. Table 2 also reports the proportion of dead cells, suggesting that apoptotic effects and antiproliferative activity are not associated with higher cytotoxicity.

Table 2 Pro-apoptotic effects of the Pd-complexes on A2780 and SKOV-3 cell lines, detected at two different concentrations (C-: untreated cells)

\begin{tabular}{|c|c|c|c|c|}
\hline Complex & $\begin{array}{l}\text { Total apoptosis (\%) } \\
\text { A2780 }\end{array}$ & $\begin{array}{l}\text { Dead cells (\%) } \\
\text { A2780 }\end{array}$ & $\begin{array}{l}\text { Total apoptosis (\%) } \\
\text { SKOV-3 }\end{array}$ & $\begin{array}{l}\text { Dead cells (\%) } \\
\text { SKOV-3 }\end{array}$ \\
\hline $\mathrm{C}-$ & 2.61 & 1.96 & 5.30 & 0.50 \\
\hline Cisplatin & $38.25(2.5 \mu \mathrm{M})$ & $3.30(2.5 \mu \mathrm{M})$ & $13.33(5 \mu \mathrm{M})$ & $1.17(5 \mu \mathrm{M})$ \\
\hline \multirow[t]{2}{*}[\mathrm{Pd}(\mu-\mathrm{Cl})(\eta^{3}-\mathrm{C}_{3}\mathrm{H}_{5})]{$_{2}$} & $8.15(1 \mu \mathrm{M})$ & $2.00(1 \mu \mathrm{M})$ & $6.55(1 \mu \mathrm{M})$ & $0.55(1 \mu \mathrm{M})$ \\
\hline & $29.40(10 \mu \mathrm{M})$ & $1.00(10 \mu \mathrm{M})$ & $7.10(10 \mu \mathrm{M})$ & $2.20(10 \mu \mathrm{M})$ \\
\hline \multirow[t]{2}{*}{$4 a$} & $3.80(1 \mu \mathrm{M})$ & $0.30(1 \mu \mathrm{M})$ & $6.35(1 \mu \mathrm{M})$ & $5.35(1 \mu \mathrm{M})$ \\
\hline & $12.35(10 \mu \mathrm{M})$ & $0.25(10 \mu \mathrm{M})$ & $12.71(10 \mu \mathrm{M})$ & $4.86(10 \mu \mathrm{M})$ \\
\hline \multirow[t]{2}{*}{$4 \mathrm{~b}$} & $8.35(1 \mu \mathrm{M})$ & $0.50(1 \mu \mathrm{M})$ & $7.25(1 \mu \mathrm{M})$ & $8.05(1 \mu \mathrm{M})$ \\
\hline & $10.66(10 \mu \mathrm{M})$ & $0.33(10 \mu \mathrm{M})$ & $44.31(10 \mu \mathrm{M})$ & $1.12(10 \mu \mathrm{M})$ \\
\hline \multirow[t]{2}{*}{$4 c$} & $54.04(0.1 \mu \mathrm{M})$ & $0.25(0.1 \mu \mathrm{M})$ & $43.78(1 \mu \mathrm{M})$ & $15.83(0.1 \mu \mathrm{M})$ \\
\hline & $98.35(1 \mu \mathrm{M})$ & $0.24(1 \mu \mathrm{M})$ & $84.04(10 \mu \mathrm{M})$ & $3.16(1 \mu \mathrm{M})$ \\
\hline \multirow[t]{2}{*}{$4 d$} & $2.25(1 \mu \mathrm{M})$ & $7.62(1 \mu \mathrm{M})$ & $6.50(0.5 \mu \mathrm{M})$ & $3.70(1 \mu \mathrm{M})$ \\
\hline & $21.00(10 \mu \mathrm{M})$ & $1.45(10 \mu \mathrm{M})$ & $52.35(5 \mu \mathrm{M})$ & $0.57(10 \mu \mathrm{M})$ \\
\hline \multirow[t]{2}{*}{$5 a$} & $3.00(1 \mu \mathrm{M})$ & $0.30(1 \mu \mathrm{M})$ & $2.45(1 \mu \mathrm{M})$ & $0.10(1 \mu \mathrm{M})$ \\
\hline & $15.25(10 \mu \mathrm{M})$ & $0.00(10 \mu \mathrm{M})$ & $3.10(10 \mu \mathrm{M})$ & $0.65(10 \mu \mathrm{M})$ \\
\hline \multirow[t]{2}{*}{$5 \mathbf{b}$} & $4.25(1 \mu \mathrm{M})$ & $0.80(1 \mu \mathrm{M})$ & $3.00(1 \mu \mathrm{M})$ & $0.45(1 \mu \mathrm{M})$ \\
\hline & $7.05(10 \mu \mathrm{M})$ & $0.15(10 \mu \mathrm{M})$ & $3.65(10 \mu \mathrm{M})$ & $0.65(10 \mu \mathrm{M})$ \\
\hline \multirow[t]{2}{*}{$5 d$} & $5.49(1 \mu \mathrm{M})$ & $2.32(1 \mu \mathrm{M})$ & $9.82(50 \mu \mathrm{M})$ & $3.06(1 \mu \mathrm{M})$ \\
\hline & $5.76(10 \mu \mathrm{M})$ & $1.43(10 \mu \mathrm{M})$ & $27.49(100 \mu \mathrm{M})$ & $1.71(10 \mu \mathrm{M})$ \\
\hline \multirow[t]{2}{*}{$6 a$} & $9.50(1 \mu \mathrm{M})$ & $0.60(1 \mu \mathrm{M})$ & $15.05(1 \mu \mathrm{M})$ & $0.60(1 \mu \mathrm{M})$ \\
\hline & $9.20(10 \mu \mathrm{M})$ & $0.15(10 \mu \mathrm{M})$ & $34.55(10 \mu \mathrm{M})$ & $0.15(10 \mu \mathrm{M})$ \\
\hline \multirow[t]{2}{*}{$6 \mathbf{b}$} & $26.52(1 \mu \mathrm{M})$ & $0.59(1 \mu \mathrm{M})$ & $6.26(1 \mu \mathrm{M})$ & $0.59(1 \mu \mathrm{M})$ \\
\hline & $61.18(10 \mu \mathrm{M})$ & $0.18(10 \mu \mathrm{M})$ & $69.10(10 \mu \mathrm{M})$ & $0.18(10 \mu \mathrm{M})$ \\
\hline \multirow[t]{2}{*}{ 6d } & $1.40(1 \mu \mathrm{M})$ & $8.92(1 \mu \mathrm{M})$ & $3.45(1 \mu \mathrm{M})$ & $0.90(1 \mu \mathrm{M})$ \\
\hline & $79.45(10 \mu \mathrm{M})$ & $0.34(10 \mu \mathrm{M})$ & $52.95(10 \mu \mathrm{M})$ & $0.15(10 \mu \mathrm{M})$ \\
\hline \multirow[t]{2}{*}{$7 a$} & $3.20(50 \mu \mathrm{M})$ & $2.10(50 \mu \mathrm{M})$ & $12.55(50 \mu \mathrm{M})$ & $0.36(50 \mu \mathrm{M})$ \\
\hline & $2.21(100 \mu \mathrm{M})$ & $1.31(100 \mu \mathrm{M})$ & $44.96(100 \mu \mathrm{M})$ & $0.29(100 \mu \mathrm{M})$ \\
\hline \multirow[t]{2}{*}{$7 \mathbf{b}$} & $2.66(1 \mu \mathrm{M})$ & $2.26(1 \mu \mathrm{M})$ & $11.25(25 \mu \mathrm{M})$ & $0.15(1 \mu \mathrm{M})$ \\
\hline & $1.60(10 \mu \mathrm{M})$ & $1.30(10 \mu \mathrm{M})$ & $53.01(50 \mu \mathrm{M})$ & $0.25(10 \mu \mathrm{M})$ \\
\hline \multirow[t]{2}{*}{$7 d$} & $2.97(1 \mu \mathrm{M})$ & $0.86(1 \mu \mathrm{M})$ & $8.73(50 \mu \mathrm{M})$ & $0.51(1 \mu \mathrm{M})$ \\
\hline & $5.65(10 \mu \mathrm{M})$ & $0.96(10 \mu \mathrm{M})$ & $36.37(100 \mu \mathrm{M})$ & $0.34(10 \mu \mathrm{M})$ \\
\hline \multirow[t]{2}{*}{$8 \mathbf{a}$} & $2.65(1 \mu \mathrm{M})$ & $0.20(1 \mu \mathrm{M})$ & $3.65(1 \mu \mathrm{M})$ & $0.35(1 \mu \mathrm{M})$ \\
\hline & $3.50(10 \mu \mathrm{M})$ & $0.25(10 \mu \mathrm{M})$ & $30.89(10 \mu \mathrm{M})$ & $0.46(10 \mu \mathrm{M})$ \\
\hline \multirow[t]{2}{*}{$8 b$} & $5.69(1 \mu \mathrm{M})$ & $1.86(1 \mu \mathrm{M})$ & $6.32(50 \mu \mathrm{M})$ & $0.41(1 \mu \mathrm{M})$ \\
\hline & $43.03(10 \mu \mathrm{M})$ & $0.34(10 \mu \mathrm{M})$ & $56.60(100 \mu \mathrm{M})$ & $0.44(10 \mu \mathrm{M})$ \\
\hline \multirow[t]{2}{*}{$8 c$} & $1.39(1 \mu \mathrm{M})$ & $7.28(1 \mu \mathrm{M})$ & $7.84(50 \mu \mathrm{M})$ & $0.39(1 \mu \mathrm{M})$ \\
\hline & $23.66(10 \mu \mathrm{M})$ & $3.09(10 \mu \mathrm{M})$ & $52.01(100 \mu \mathrm{M})$ & $0.38(10 \mu \mathrm{M})$ \\
\hline \multirow[t]{2}{*}{ 8d } & $5.90(1 \mu \mathrm{M})$ & $0.56(1 \mu \mathrm{M})$ & $12.74(25 \mu \mathrm{M})$ & $1.29(1 \mu \mathrm{M})$ \\
\hline & $61.17(10 \mu \mathrm{M})$ & $0.00(10 \mu \mathrm{M})$ & $79.32(50 \mu \mathrm{M})$ & $3.18(10 \mu \mathrm{M})$ \\
\hline
\end{tabular}




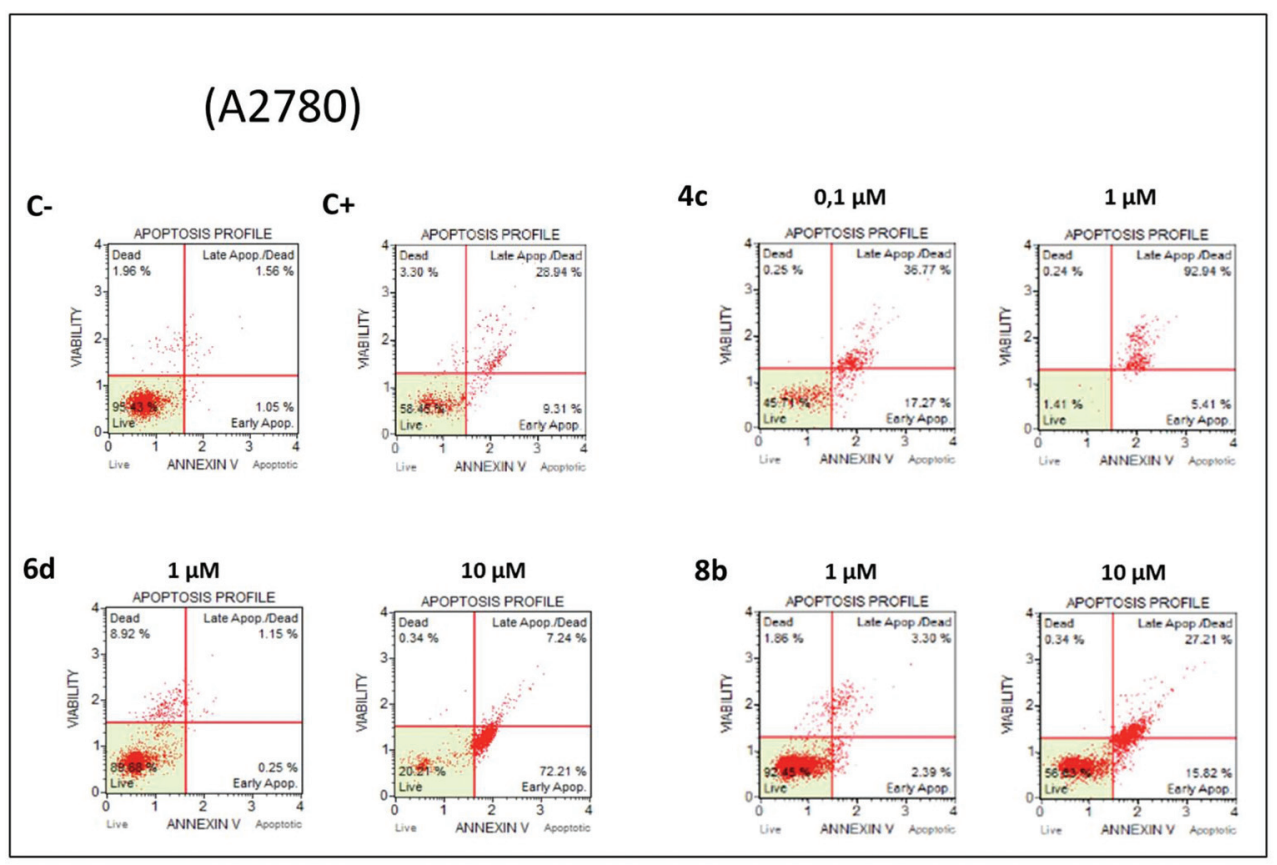

Fig. 3 Representative apoptosis profiles of A2780 cells untreated (C-), treated with cisplatin (C+) and treated with complexes $4 \mathrm{c}$, $6 \mathrm{~d}$ and $\mathbf{8 b}$ $(110 \mu \mathrm{M})$ for $72 \mathrm{~h}$.

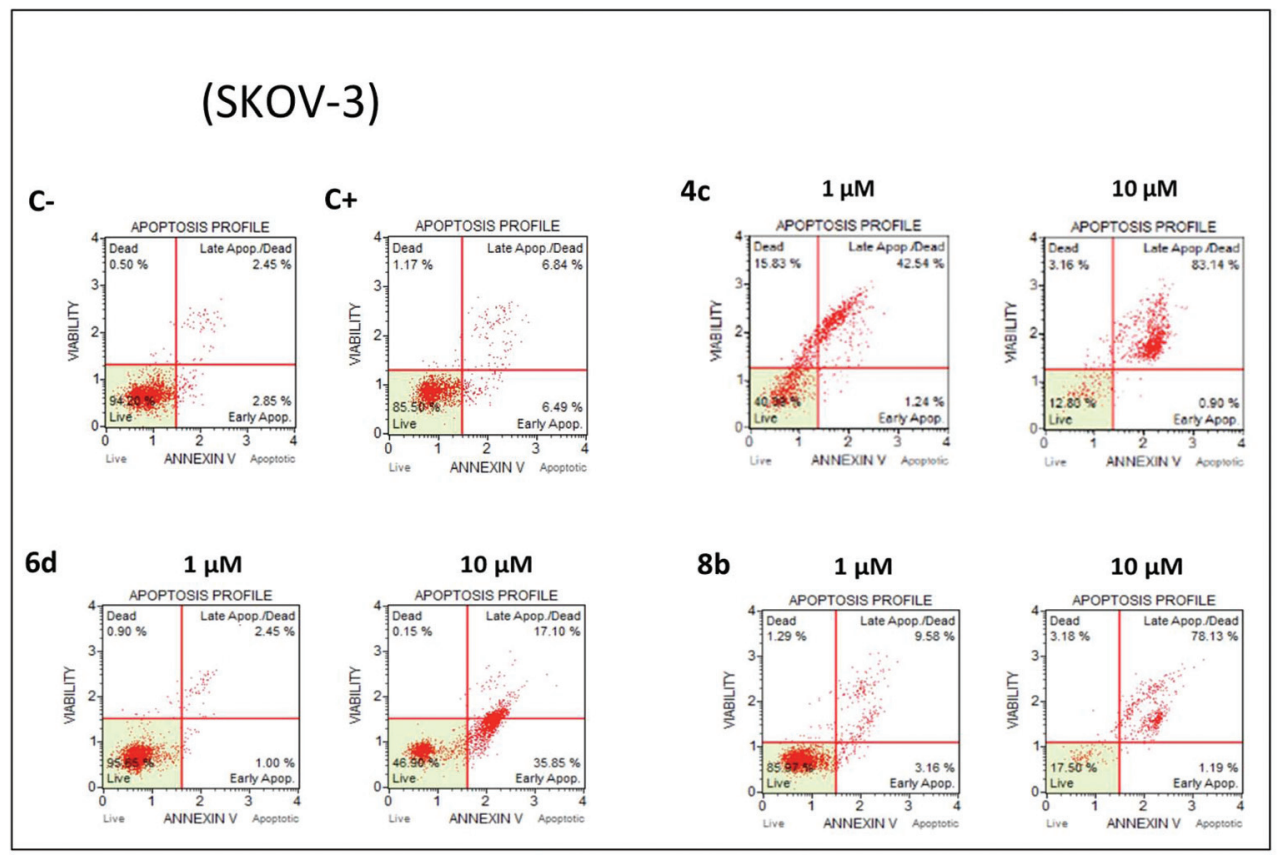

Fig. 4 Representative apoptosis profiles of SKOV-3 cells untreated (C-), treated with cisplatin $\left(C_{+}\right)$and treated with complexes $4 c, 6 d$ and $8 b$ $(1-10 \mu \mathrm{M})$ for $72 \mathrm{~h}$.

\section{Conclusions}

In this contribution we have proposed a complete and optimised protocol for the synthesis of new cationic allyl palladium complexes bearing NHC ligands with a purinic frame- work. The combination of the palladium-allyl organometallic function with the natural imprint of the spectator ligand seems to play a crucial role in promoting the cytotoxic properties of these compounds. Their antiproliferative activity has been tested in vitro on cisplatin-sensitive A2780 and cisplatin- 
resistant SKOV-3 cell lines and compared with cisplatin as an etalon drug. Complexes $\mathbf{4 c}$, $\mathbf{4 d}$, and 5d (for A2780 cells) and $\mathbf{4 d}$ (for SKOV-3 cells) displayed higher antiproliferative effects than cisplatin, showing that the simultaneous coordination of one triphenylphosphine and one $\mathrm{N}$-heterocyclic carbene on the palladium allyl fragment represents the most promising configuration. However, the most active compounds on cancer cells are almost inactive on normal cells, suggesting a marked cancer selectivity.

Moreover, it was proved that the anti-proliferative activity of many of the synthesized complexes is associated with induction of apoptosis. No major cytotoxicity was observed after evaluation of the proportion of dead cells (Table 2).

Despite the fact that our data do not clarify the molecular target(s) of the compounds presented here, the apoptosis network should be considered as a major cellular target.

A future extension of this first encouraging study will have as its main goals the identification of the true molecular target of these allyl palladium complexes and the definition of their mode of action. As a further possible application these compounds might be considered for combined antitumor therapy after co-administration with pro-apoptotic molecules exhibiting a verified mechanism of action (paclitaxel, topotecan, doxorubicin, etoposide, or gemcitabine, or with antineoplastic molecules displaying different mechanisms of action). ${ }^{33-41} \mathrm{~A}$ recognized advantage of the combined antitumor approach is the expected fewer side effects associated with the use of minor concentrations. In any case, in vivo activity of the most efficient compounds studied here would clarify whether the described effects on tumor cell lines is accompanied by the absence of side effects on normal cells and networks. Only in this case these complexes might be further considered for possible biomedical application in the field of anticancer treatment.

\section{Experimental}

All the synthesis of the complexes were carried out using standard Schlenk techniques under an atmosphere of dry nitrogen. Solvents were dried and distilled according to standard methods: $\mathrm{CH}_{2} \mathrm{Cl}_{2}$ was first treated with $3 \AA$ molecular sieves and then distilled over $\mathrm{P}_{2} \mathrm{O}_{5} ; \mathrm{CH}_{3} \mathrm{CN}$ was distilled over $\mathrm{CaH}_{2}$ and stored over $3 \AA$ molecular sieves. All the other chemicals were commercially available grade products and were used as purchased.

Complexes $\mathbf{3 a}, \mathbf{3} \mathbf{b}$, and $\mathbf{3 d}$ and complexes $\mathbf{7 a}, \mathbf{7} \mathbf{b}$, and $\mathbf{7 d}$ were synthesized according to the procedure described in a previous study. ${ }^{19}$

The IR, ${ }^{1} \mathrm{H},{ }^{13} \mathrm{C}$ and ${ }^{31} \mathrm{P}$ NMR spectra were recorded on a PerkinElmer Spectrum One spectrophotometer and a Bruker 300 or $400 \mathrm{MHz}$ Avance spectrometer, respectively.

Elemental analysis was carried out using an Elemental CHN "CUBO Micro Vario” analyzer.

ESI-MS analyses were performed using a LCQ-Duo (Thermo Finnigan) operating in the positive ion mode. Instrumental parameters: capillary voltage $10 \mathrm{~V}$, spray voltage $4.5 \mathrm{kV}$, capil- lary temperature $200{ }^{\circ} \mathrm{C}$, mass scan range 150 to $2000 \mathrm{amu}, \mathrm{N}_{2}$ was used as the sheath gas and the He pressure inside the trap was kept constant.

The pressure directly read by an ion gauge (in the absence of a $\mathrm{N}_{2}$ stream) was $1.33 \times 10^{-5}$ Torr. Sample solutions were prepared by dissolving the compounds in acetonitrile and directly infusing into the ESI source with a syringe pump at $8 \mu \mathrm{L} \min ^{-1}$ flow rate.

\section{General procedure for the synthesis of alkyl-functionalized caffeine, theophylline and theobromine (1)}

$5.55 \mathrm{mmol}$ ( $c a .1 \mathrm{~g}$ ) of the starting xanthine (theophylline or theobromine) and $1.15 \mathrm{~g}(8.33 \mathrm{mmol})$ of $\mathrm{K}_{2} \mathrm{CO}_{3}$ were suspended in $20 \mathrm{~mL}$ of DMF. Two equivalents $(11.1 \mathrm{mmol})$ of the appropriate alkyl halide were added to the suspension and the resulting mixture was vigorously stirred overnight at R.T. Finally, the compounds were precipitated by the addition of $\mathrm{H}_{2} \mathrm{O}($ ca. $100 \mathrm{~mL})$.

\section{7-Benzyl-1,3-dimethylxanthine (1b)}

White solid, yield 93\%.

${ }^{1} \mathrm{H}-\mathrm{NMR}\left(400 \mathrm{MHz}, \mathrm{CDCl}_{3}, T=298 \mathrm{~K}, \mathrm{ppm}\right) \delta: 3.43(\mathrm{~s}, 3 \mathrm{H}$, $\left.\mathrm{N}-\mathrm{CH}_{3}\right), 3.60\left(\mathrm{~s}, 3 \mathrm{H}, \mathrm{N}-\mathrm{CH}_{3}\right), 5.52\left(\mathrm{~s}, 2 \mathrm{H}, \mathrm{N}-\mathrm{CH}_{2}\right), 7.30-7.40$ (m, 5H, Ph), 7.58 (s, 1H, NCHN). ${ }^{13} \mathrm{C}\left\{{ }^{1} \mathrm{H}\right\}-\mathrm{NMR}\left(\mathrm{CDCl}_{3}, T=\right.$ $298 \mathrm{~K}, \mathrm{ppm}) \delta: 28.0\left(\mathrm{~N}-\mathrm{CH}_{3}\right), 29.8\left(\mathrm{~N}-\mathrm{CH}_{3}\right), 50.3\left(\mathrm{~N}-\mathrm{CH}_{2}\right)$, $107.0\left(\mathrm{C}^{5}\right), 128.0,128.7,129.1,135.4\left(\mathrm{C}_{\mathrm{Ph}}\right), 140.9(\mathrm{~N}-\mathrm{CH}-\mathrm{N})$, $148.9\left(\mathrm{C}^{4}\right), 151.7(\mathrm{C}=\mathrm{O}), 155.3(\mathrm{C}=\mathrm{O})$.

ESI-MS $\left(\mathrm{CH}_{3} \mathrm{CN}\right): m / z$ calcd for $\mathrm{C}_{14} \mathrm{H}_{15} \mathrm{~N}_{4} \mathrm{O}_{2}[\mathrm{M}+\mathrm{H}]^{+} 271.12$; found: 271.06 .

Anal. calcd for $\mathrm{C}_{14} \mathrm{H}_{14} \mathrm{~N}_{4} \mathrm{O}_{2}$ : C 62.21, $\mathrm{H}$ 5.22, N 20.73. Found: C 62.32, H 5.14, N 20.64.

\section{7-Phenylpropargyl-1,3-dimethylxanthine (1c)}

Pink solid, yield 95\%.

${ }^{1} \mathrm{H}-\mathrm{NMR}\left(400 \mathrm{MHz}, \mathrm{CDCl}_{3}, T=298 \mathrm{~K}, \mathrm{ppm}\right) \delta: 3.45(\mathrm{~s}, 3 \mathrm{H}$, $\left.\mathrm{N}-\mathrm{CH}_{3}\right), 3.63\left(\mathrm{~s}, 3 \mathrm{H}, \mathrm{N}-\mathrm{CH}_{3}\right), 5.42\left(\mathrm{~s}, 2 \mathrm{H}, \mathrm{N}-\mathrm{CH}_{2}\right), 7.32-7.50$ (m, 5H, Ph), 7.94 (s, 1H, NCHN).

${ }^{13} \mathrm{C}\left\{{ }^{1} \mathrm{H}\right\}$-NMR $\left(\mathrm{CDCl}_{3}, T=298 \mathrm{~K}, \mathrm{ppm}\right) \delta: 28.0\left(\mathrm{~N}-\mathrm{CH}_{3}\right), 29.8$ $\left(\mathrm{N}-\mathrm{CH}_{3}\right), 37.4\left(\mathrm{~N}-\mathrm{CH}_{2}\right), 80.5\left(\equiv \underline{\mathrm{C}}-\mathrm{CH}_{2}\right), 87.6$ ( $\left.\equiv \underline{\mathrm{C}}-\mathrm{Ph}\right), 106.8$ $\left(\mathrm{C}^{5}\right), 121.5,128.5,129.2,131.9\left(\mathrm{C}_{\mathrm{Ph}}\right), 140.6(\mathrm{~N}-\mathrm{CH}-\mathrm{N}), 148.9$ $\left(\mathrm{C}^{4}\right), 151.7(\mathrm{C}=\mathrm{O}), 155.3(\mathrm{C}=\mathrm{O})$.

ESI-MS $\left(\mathrm{CH}_{3} \mathrm{CN}\right): m / z$ calcd for $\mathrm{C}_{16} \mathrm{H}_{15} \mathrm{~N}_{4} \mathrm{O}_{2}[\mathrm{M}+\mathrm{H}]^{+} 295.12$; found: 294.98.

Anal. calcd for $\mathrm{C}_{16} \mathrm{H}_{14} \mathrm{~N}_{4} \mathrm{O}_{2}$ : C 65.30, H 4.79, N 19.04 . Found: C 65.49, H 4.83, N 18.89.

\section{1-Benzyl-3,7-dimethylxanthine (1d)}

White solid, yield 95\%.

${ }^{1} \mathrm{H}-\mathrm{NMR}\left(400 \mathrm{MHz}, \mathrm{CDCl}_{3}, T=298 \mathrm{~K}, \mathrm{ppm}\right) \delta: 3.59$ (s, 3H, $\left.\mathrm{N}-\mathrm{CH}_{3}\right), 4.00\left(\mathrm{~s}, 3 \mathrm{H}, \mathrm{N}-\mathrm{CH}_{3}\right), 5.22\left(\mathrm{~s}, 2 \mathrm{H}, \mathrm{N}-\mathrm{CH}_{2}\right), 7.25-7.55$ (m, 5H, Ph), 7.51 (s, 1H, NCHN). ${ }^{13} \mathrm{C}\left\{{ }^{1} \mathrm{H}\right\}-\mathrm{NMR}\left(\mathrm{CDCl}_{3}, T=\right.$ $298 \mathrm{~K}, \mathrm{ppm}) \delta: 29.8\left(\mathrm{~N}-\mathrm{CH}_{3}\right), 33.6\left(\mathrm{~N}-\mathrm{CH}_{3}\right), 44.5\left(\mathrm{~N}-\mathrm{CH}_{2}\right)$, $107.7\left(\mathrm{C}^{5}\right), 127.5,128.4,128.8,137.3\left(\mathrm{C}_{\mathrm{Ph}}\right), 141.5(\mathrm{~N}-\mathrm{CH}-\mathrm{N})$, $148.9\left(\mathrm{C}^{4}\right), 151.6(\mathrm{C}=\mathrm{O}), 155.3(\mathrm{C}=\mathrm{O})$.

ESI-MS $\left(\mathrm{CH}_{3} \mathrm{CN}\right): m / z$ calcd for $\mathrm{C}_{14} \mathrm{H}_{15} \mathrm{~N}_{4} \mathrm{O}_{2}[\mathrm{M}+\mathrm{H}]^{+} 271.12$; found: 271.14 . 
Anal. calcd for $\mathrm{C}_{14} \mathrm{H}_{14} \mathrm{~N}_{4} \mathrm{O}_{2}$ : C 62.21, H 5.22, N 20.73. Found: C 62.45, H 5.07, N 20.99.

General procedure for the synthesis of the imidazolium salts from functionalized theophylline and theobromine (2)

Into a $50 \mathrm{~mL}$ flask, $1.8 \mathrm{mmol}$ of functionalized xanthine (1a-d where 1a $=$ commercial caffeine) were dissolved in $c a .25 \mathrm{~mL}$ of $\mathrm{CH}_{3} \mathrm{CN}$. After the addition of one equivalent $(1.8 \mathrm{mmol})$ of $\mathrm{Me}_{3} \mathrm{OBF}_{4}$ the resulting solution was vigorously stirred for $5 \mathrm{~min}$ and then $100 \mathrm{mg}$ of $\mathrm{Na}_{2} \mathrm{CO}_{3}$ were added to the mixture, which was stirred for $45 \mathrm{~min}$. Further addition of 0.6 equivalent $(1.1 \mathrm{mmol})$ of $\mathrm{Me}_{3} \mathrm{OBF}_{4}$ and $10 \mathrm{~min}$ of additional stirring led to virtual completion. The excess of base $\mathrm{Na}_{2} \mathrm{CO}_{3}$ and the $\mathrm{NaBF}_{4}$ formed were filtered off and the solvent was completely removed under reduced pressure. The solid was washed with three aliquots of a $2: 1$ mixture of $\mathrm{Et}_{2} \mathrm{O} / \mathrm{CH}_{2} \mathrm{Cl}_{2}$ on a sintered glass filter, dried under vacuum and characterized.

\section{1,3,7,9-Tetramethylxanthinium tetrafluoroborate (2a)}

White solid, yield 97\%, m.p. $=132-133^{\circ} \mathrm{C}$.

${ }^{1} \mathrm{H}-\mathrm{NMR}\left(400 \mathrm{MHz}, \mathrm{CD}_{3} \mathrm{CN}, T=298 \mathrm{~K}, \mathrm{ppm}\right) \delta: 3.35(\mathrm{~s}, 3 \mathrm{H}$, $\left.\mathrm{N}-\mathrm{CH}_{3}\right), 3.74\left(\mathrm{~s}, 3 \mathrm{H}, \mathrm{N}-\mathrm{CH}_{3}\right), 4.08\left(\mathrm{~s}, 6 \mathrm{H}, 2 \mathrm{~N}-\mathrm{CH}_{3}\right), 8.47(\mathrm{~s}, 1 \mathrm{H}$, NCHN). ${ }^{13} \mathrm{C}\left\{{ }^{1} \mathrm{H}\right\}-\mathrm{NMR}\left(\mathrm{CD}_{3} \mathrm{CN}, \quad T=298 \mathrm{~K}, \mathrm{ppm}\right) \delta: 28.7$ $\left(\mathrm{N}-\mathrm{CH}_{3}\right), 31.8\left(\mathrm{~N}-\mathrm{CH}_{3}\right), 36.3\left(\mathrm{~N}-\mathrm{CH}_{3}\right), 37.6\left(\mathrm{~N}-\mathrm{CH}_{3}\right), 109.1\left(\mathrm{C}^{5}\right)$, $139.3(\mathrm{~N}-\mathrm{CH}-\mathrm{N}), 140.2\left(\mathrm{C}^{4}\right), 151.1(\mathrm{C}=\mathrm{O}), 154.2(\mathrm{C}=\mathrm{O})$.

ESI-MS $\left(\mathrm{CH}_{3} \mathrm{CN}\right): \mathrm{m} / \mathrm{z}$ calcd for $\mathrm{C}_{9} \mathrm{H}_{13} \mathrm{~N}_{4} \mathrm{O}_{2}[\mathrm{M}]^{+}$209.10; found: 209.07 .

Anal. calcd for $\mathrm{C}_{9} \mathrm{H}_{13} \mathrm{BF}_{4} \mathrm{~N}_{4} \mathrm{O}_{2}$ : C 36.52, $\mathrm{H}$ 4.43, N 18.93 . Found: C 36.74, H 4.61, N 18.88.

\section{7-Benzyl-1,3,9-trimethylxanthinium tetrafluoroborate (2b)}

White solid, yield 91\%, m.p. $=251-253{ }^{\circ} \mathrm{C}$.

${ }^{1} \mathrm{H}-\mathrm{NMR}\left(400 \mathrm{MHz}, \mathrm{CD}_{3} \mathrm{CN}, T=298 \mathrm{~K}, \mathrm{ppm}\right) \delta: 3.33(\mathrm{~s}, 3 \mathrm{H}$, $\left.\mathrm{N}-\mathrm{CH}_{3}\right), 3.73\left(\mathrm{~s}, 3 \mathrm{H}, \mathrm{N}-\mathrm{CH}_{3}\right), 4.06\left(\mathrm{~s}, 3 \mathrm{H}, \mathrm{N}-\mathrm{CH}_{3}\right), 5.70(\mathrm{~s}, 2 \mathrm{H}$, $\mathrm{N}-\mathrm{CH}_{2}$ ), 7.45-7.48 (m, 5H, Ph), 8.56 (s, 1H, NCHN). ${ }^{13} \mathrm{C}\left\{{ }^{1} \mathrm{H}\right\}-$ NMR ( $\left.\mathrm{CD}_{3} \mathrm{CN}, T=298 \mathrm{~K}, \mathrm{ppm}\right) \delta: 28.8\left(\mathrm{~N}-\mathrm{CH}_{3}\right), 31.8\left(\mathrm{~N}-\mathrm{CH}_{3}\right)$, $37.9\left(\mathrm{~N}-\mathrm{CH}_{3}\right), 52.7\left(\mathrm{~N}-\mathrm{CH}_{2}\right), 108.4\left(\mathrm{C}^{5}\right), 129.2,129.7,129.8$, $133.9\left(\mathrm{C}_{\mathrm{Ph}}\right), 138.9(\mathrm{~N}-\mathrm{CH}-\mathrm{N}), 140.6\left(\mathrm{C}^{4}\right), 151.0(\mathrm{C}=\mathrm{O}), 154.0$ $(\mathrm{C}=\mathrm{O})$.

ESI-MS $\left(\mathrm{CH}_{3} \mathrm{CN}\right): \mathrm{m} / \mathrm{z}$ calcd for $\mathrm{C}_{15} \mathrm{H}_{17} \mathrm{~N}_{4} \mathrm{O}_{2}[\mathrm{M}]^{+}$285.13; found: 285.04 .

Anal. calcd for $\mathrm{C}_{15} \mathrm{H}_{17} \mathrm{BF}_{4} \mathrm{~N}_{4} \mathrm{O}_{2}$ : C 48.41, $\mathrm{H}$ 4.60, N 15.06. Found: C 48.39, H 4.64, N 15.18.

\section{7-Phenylpropargyl-1,3,9-trimethylxanthinium tetrafluoroborate} (2c)

White solid, yield 92\% (612 mg), m.p. $=231 \mathrm{dec} .{ }^{\circ} \mathrm{C}$.

${ }^{1} \mathrm{H}-\mathrm{NMR}\left(400 \mathrm{MHz}, \mathrm{CD}_{3} \mathrm{CN}, T=298 \mathrm{~K}, \mathrm{ppm}\right) \delta: 3.37(\mathrm{~s}, 3 \mathrm{H}$, $\left.\mathrm{N}-\mathrm{CH}_{3}\right), 3.76\left(\mathrm{~s}, 3 \mathrm{H}, \mathrm{N}-\mathrm{CH}_{3}\right), 4.14\left(\mathrm{~s}, 3 \mathrm{H}, \mathrm{N}-\mathrm{CH}_{3}\right), 5.57(\mathrm{~s}, 2 \mathrm{H}$, $\mathrm{N}-\mathrm{CH}_{2}$ ), 7.42-7.60 (m, 5H, Ph), 8.85 (s, 1H, NCHN). ${ }^{13} \mathrm{C}\left\{{ }^{1} \mathrm{H}\right\}-$ NMR ( $\left.\mathrm{CD}_{3} \mathrm{CN}, T=298 \mathrm{~K}, \mathrm{ppm}\right) \delta: 28.8\left(\mathrm{~N}-\mathrm{CH}_{3}\right), 31.9\left(\mathrm{~N}-\mathrm{CH}_{3}\right)$, $37.9\left(\mathrm{~N}-\mathrm{CH}_{3}\right), 40.7\left(\mathrm{~N}-\mathrm{CH}_{2}\right), 79.2\left(\equiv \underline{\mathrm{C}}-\mathrm{CH}_{2}\right), 89.4$ ( $\left.\equiv \underline{\mathrm{C}}-\mathrm{Ph}\right)$, $108.3\left(\mathrm{C}^{5}\right), 121.6,129.4,130.4,132.5\left(\mathrm{C}_{\mathrm{Ph}}\right), 138.8\left(\mathrm{~N}-\overline{\mathrm{CH}}_{-} \mathrm{N}\right)$, $140.6\left(\mathrm{C}^{4}\right), 151.0(\mathrm{C}=\mathrm{O}), 154.0(\mathrm{C}=\mathrm{O})$.

ESI-MS $\left(\mathrm{CH}_{3} \mathrm{CN}\right): \mathrm{m} / z$ calcd for $\mathrm{C}_{17} \mathrm{H}_{17} \mathrm{~N}_{4} \mathrm{O}_{2}[\mathrm{M}]^{+}$309.13; found: 309.02 .
Anal. calcd for $\mathrm{C}_{17} \mathrm{H}_{17} \mathrm{BF}_{4} \mathrm{~N}_{4} \mathrm{O}_{2}$ : C 51.54, $\mathrm{H}$ 4.33, $\mathrm{N} 14.14$. Found: C 51.73, H 4.28, N 14.31.

\section{1-Benzyl-3,7,9-trimethylxanthinium tetrafluoroborate (2d)}

White solid, yield 94\% (621 mg), m.p. $=171-172{ }^{\circ} \mathrm{C}$.

${ }^{1} \mathrm{H}-\mathrm{NMR}\left(400 \mathrm{MHz}, \mathrm{CD}_{3} \mathrm{CN}, T=298 \mathrm{~K}, \mathrm{ppm}\right) \delta: 3.74(\mathrm{~s}, 3 \mathrm{H}$, $\left.\mathrm{N}-\mathrm{CH}_{3}\right), 4.08\left(\mathrm{~s}, 3 \mathrm{H}, \mathrm{N}-\mathrm{CH}_{3}\right), 4.09\left(\mathrm{~s}, 3 \mathrm{H}, \mathrm{N}-\mathrm{CH}_{3}\right), 5.17(\mathrm{~s}, 2 \mathrm{H}$, $\mathrm{N}-\mathrm{CH}_{2}$ ), 7.28-7.42 (m, 5H, Ph), $8.48(\mathrm{~s}, 1 \mathrm{H}, \mathrm{NCHN}) .{ }^{13} \mathrm{C}\left\{{ }^{1} \mathrm{H}\right\}-$ NMR $\left(\mathrm{CD}_{3} \mathrm{CN}, T=298 \mathrm{~K}, \mathrm{ppm}\right) \delta: 31.9\left(\mathrm{~N}-\mathrm{CH}_{3}\right), 36.4\left(\mathrm{~N}-\mathrm{CH}_{3}\right)$, $37.6\left(\mathrm{~N}-\mathrm{CH}_{3}\right), 45.6\left(\mathrm{~N}-\mathrm{CH}_{2}\right), 109.2\left(\mathrm{C}^{5}\right), 128.3,128.6,129.1$, $137.1\left(\mathrm{C}_{\mathrm{Ph}}\right), 139.4(\mathrm{~N}-\mathrm{CH}-\mathrm{N}), 140.5\left(\mathrm{C}^{4}\right), 151.0(\mathrm{C}=\mathrm{O}), 154.1$ $(\mathrm{C}=\mathrm{O})$.

ESI-MS $\left(\mathrm{CH}_{3} \mathrm{CN}\right): \mathrm{m} / z$ calcd for $\mathrm{C}_{15} \mathrm{H}_{17} \mathrm{~N}_{4} \mathrm{O}_{2}[\mathrm{M}]^{+}$285.13; found: 285.11 .

Anal. calcd for $\mathrm{C}_{15} \mathrm{H}_{17} \mathrm{BF}_{4} \mathrm{~N}_{4} \mathrm{O}_{2}$ : C 48.41, $\mathrm{H}$ 4.60, N 15.06. Found: C 48.65, H 4.72, N 15.14.

\section{Preparation of a 1:1 mixture of $3 \mathrm{c}$ and $\mathrm{AgBF}_{4}$}

$0.1130 \mathrm{~g}(0.2852 \mathrm{mmol})$ of the imidazolium salt $2 \mathrm{c}$ was dissolved in $30 \mathrm{~mL}$ of anhydrous $\mathrm{CH}_{3} \mathrm{CN}$ in a $100 \mathrm{~mL}$ two necked flask and $0.09364 \mathrm{~g}(0.1571 \mathrm{mmol})$ of $\mathrm{Ag}_{2} \mathrm{O}$ was added under an inert atmosphere (Ar).

The mixture was stirred for $24 \mathrm{~h}$ at R.T. in the dark. The solution was filtered on a Millipore membrane filter in order to remove excess $\mathrm{Ag}_{2} \mathrm{O}$. The resulting clear solution was concentrated under vacuum and the title complex was precipitated by the addition of diethyl ether. The white complex was separated by filtration and repeatedly washed with diethyl ether and $n$-pentane and finally dried under vacuum.

$0.1305 \mathrm{~g}$ (yield 91\%) of a $1: 1$ mixture of $3 \mathrm{c}$ and $\mathrm{AgBF}_{4}$ was obtained.

${ }^{1} \mathrm{H}-\mathrm{NMR}\left(400 \mathrm{MHz}, \mathrm{CD}_{3} \mathrm{CN}, T=298 \mathrm{~K}, \mathrm{ppm}\right) \delta: 3.35$ (s, 6H, $\left.2 \mathrm{NCH}_{3}\right), 3.70\left(\mathrm{~s}, 6 \mathrm{H}, 2 \mathrm{NCH}_{3}\right), 4.03\left(\mathrm{~s}, 6 \mathrm{H}, 2 \mathrm{NCH}_{3}\right), 5.40(\mathrm{~s}, 4 \mathrm{H}$, $\left.2 \mathrm{NCH}_{2}\right), 7.27-7.43$ (m, 10H, 2Ph). ${ }^{13} \mathrm{C}\left\{{ }^{1} \mathrm{H}\right\}-\mathrm{NMR}\left(\mathrm{CD}_{3} \mathrm{CN}, T=\right.$ $298 \mathrm{~K}, \mathrm{ppm}) \delta: 27.9\left(\mathrm{CH}_{3}, \mathrm{NCH}_{3}\right), 31.4\left(\mathrm{CH}_{3}, \mathrm{NCH}_{3}\right), 40.1\left(\mathrm{CH}_{2}\right.$, $\left.\mathrm{NCH}_{2}\right), 40.2\left(\mathrm{CH}_{3}, \mathrm{NCH}_{3}\right), 82.5\left(\mathrm{C}, \mathrm{CH}_{2}-\mathrm{C} \equiv\right), 88.7$ (C, Ph- $\left.\underline{\mathrm{C}} \equiv\right)$, 108.7 (C, C $\left.{ }^{5}\right), 121.3-131.8(\mathrm{Ph}), 140.4\left(\mathrm{C}, \mathrm{C}^{4}\right), 150.7(\mathrm{C}, \mathrm{C}=\mathrm{O})$, $153.5(\mathrm{C}, \mathrm{C}=\mathrm{O}), 187.1$ (C, carbene).

IR $(\mathrm{KBr}): \nu_{\mathrm{C} \equiv \mathrm{C}}=2218 \mathrm{~cm}^{-1}, \nu_{\mathrm{CO}}=1710,1668 \mathrm{~cm}^{-1}, \nu_{\mathrm{BF}}=$ $1054 \mathrm{~cm}^{-1}$.

Anal. calcd for $\mathrm{C}_{34} \mathrm{H}_{32} \mathrm{Ag}_{2} \mathrm{~B}_{2} \mathrm{~F}_{8} \mathrm{~N}_{8} \mathrm{O}_{4}: \mathrm{C}$ 40.59, $\mathrm{H}$ 3.21, N 11.14. Found: C 40.82, H 3.04, N 11.35.

\section{Synthesis of complex $4 a$}

$0.0195 \mathrm{~g}(0.053 \mathrm{mmol})$ of the dimer $\left[\mathrm{Pd}(\mu-\mathrm{Cl})\left(\eta^{3}-\mathrm{C}_{3} \mathrm{H}_{5}\right)\right]_{2}$ and $0.0279 \mathrm{~g}(0.106 \mathrm{mmol})$ of $\mathrm{PPh}_{3}$ were dissolved in $c a .20 \mathrm{~mL}$ of anhydrous $\mathrm{CH}_{3} \mathrm{CN}$ in a $50 \mathrm{~mL}$ two necked flask under an inert atmosphere $(\mathrm{Ar})$. The resulting mixture was treated with $0.0388 \mathrm{~g}(0.048 \mathrm{mmol})$ of $3 \mathrm{a} / \mathrm{AgBF}_{4}$ and stirred at RT for $c a$. $15 \mathrm{~min}$.

The precipitated $\mathrm{AgCl}$ was removed by filtration on a Millipore membrane filter.

Addition of diethyl ether to the concentrated solution yielded the precipitation of complex $4 \mathbf{a}$ as a yellow solid, which was filtered off on a Gooch and washed with $n$-pentane.

$0.0576 \mathrm{~g}$ of $\mathbf{4 a}$ was obtained (yield $85 \%$ ). 
${ }^{1} \mathrm{H}-\mathrm{NMR}\left(400 \mathrm{MHz}, T=298 \mathrm{~K}, \mathrm{CDCl}_{3}, \mathrm{ppm}\right) \delta: 3.16(\mathrm{~d}, 1 \mathrm{H}$, $J=13.4 \mathrm{~Hz}$, anti allyl- $\mathrm{H}$ trans $\mathrm{C}), 3.26(\mathrm{~d}, 1 \mathrm{H}, J=13.7 \mathrm{~Hz}$, anti allyl-H trans $\mathrm{C}), 3.27(\mathrm{~m}, 1 \mathrm{H}$, anti allyl-H trans $\mathrm{P}), 3.38(\mathrm{~s}, 6 \mathrm{H}$, $\left.2 \mathrm{NCH}_{3}\right), 3.59\left(\mathrm{~s}, 6 \mathrm{H}, 2 \mathrm{NCH}_{3}\right), 3.61\left(\mathrm{~s}, 6 \mathrm{H}, 2 \mathrm{NCH}_{3}\right), 3.68(\mathrm{~m}, 1 \mathrm{H}$, anti allyl-H trans $\mathrm{P}), 3.76\left(\mathrm{~s}, 3 \mathrm{H}, \mathrm{NCH}_{3}\right), 3.78\left(\mathrm{~s}, 3 \mathrm{H}, \mathrm{NCH}_{3}\right)$, $4.11(\mathrm{~d}, 1 \mathrm{H}, J=8.4 \mathrm{~Hz}$, syn allyl-H trans $\mathrm{C}), 4.19(\mathrm{~d}, 1 \mathrm{H}, J=$ $7.6 \mathrm{~Hz}$, syn allyl- $\mathrm{H}$ trans $\mathrm{C}), 4.59\left(\mathrm{dd}, 1 \mathrm{H}, J_{\mathrm{H}-\mathrm{H}}=J_{\mathrm{H}-\mathrm{P}}=5.9 \mathrm{~Hz}\right.$, syn allyl- $\mathrm{H}$ trans $\mathrm{P}), 4.80\left(\mathrm{dt}, 1 \mathrm{H}, J_{\mathrm{H}-\mathrm{H}}=J_{\mathrm{H}-\mathrm{P}}=6.6 \mathrm{~Hz}\right.$, syn allyl$\mathrm{H}$ trans $\mathrm{P}), 5.71(\mathrm{~m}, 1 \mathrm{H}$, central allyl- $\mathrm{H}), 5.99(\mathrm{~m}, 1 \mathrm{H}$, central allyl-H), 7.25-7.53 (m, 30H, 6Ph). ${ }^{13} \mathrm{C}\left\{{ }^{1} \mathrm{H}\right\}$-NMR $(T=298 \mathrm{~K}$, $\left.\mathrm{CDCl}_{3}, \mathrm{ppm}\right) \delta: 28.6\left(\mathrm{CH}_{3}, \mathrm{NCH}_{3}\right), 31.7\left(\mathrm{CH}_{3}, \mathrm{NCH}_{3}\right), 36.9$ $\left(\mathrm{CH}_{3}, \mathrm{NCH}_{3}\right), 37.1\left(\mathrm{CH}_{3}, \mathrm{NCH}_{3}\right), 38.7\left(\mathrm{CH}_{3}, \mathrm{NCH}_{3}\right), 38.9\left(\mathrm{CH}_{3}\right.$, $\left.\mathrm{NCH}_{3}\right), 68.5\left(\mathrm{~d}, \mathrm{CH}_{2}, J_{\mathrm{C}-\mathrm{P}}=28.5 \mathrm{~Hz}\right.$, allyl trans $\left.P\right), 69.3\left(\mathrm{~d}, \mathrm{CH}_{2}\right.$, $J_{\mathrm{C}-\mathrm{P}}=27.6 \mathrm{~Hz}$, allyl trans $\left.P\right), 69.4\left(\mathrm{~d}, \mathrm{CH}_{2}, J_{\mathrm{C}-\mathrm{P}}=1.8 \mathrm{~Hz}\right.$, allyl trans C), $69.4\left(\mathrm{~d}, \mathrm{CH}_{2}, J_{\mathrm{C}-\mathrm{P}}=1.7 \mathrm{~Hz}\right.$, allyl trans $\left.C\right), 110.4\left(\mathrm{C}, \mathrm{C}^{5}\right)$, $110.6\left(\mathrm{C}, \mathrm{C}^{5}\right), 121.4\left(\mathrm{~d}, \mathrm{CH}, J_{\mathrm{C}-\mathrm{P}}=5.4 \mathrm{~Hz}\right.$, central allyl), 122.8 $\left(\mathrm{d}, \mathrm{CH}, J_{\mathrm{C}-\mathrm{P}}=5.1 \mathrm{~Hz}\right.$, central allyl), 129.2-133.2 (Ph), 141.0 $\left(\mathrm{C}, \mathrm{C}^{4}\right), 141.1\left(\mathrm{C}, \mathrm{C}^{4}\right), 150.4(\mathrm{C}, \mathrm{C}=\mathrm{O}), 153.0(\mathrm{C}, \mathrm{C}=\mathrm{O}), 185.8$ $\left(\mathrm{d}, \mathrm{C}, J_{\mathrm{C}-\mathrm{P}}=19.7 \mathrm{~Hz}\right.$, carbene), $186.2\left(\mathrm{~d}, \mathrm{C}, J_{\mathrm{C}-\mathrm{P}}=19.3 \mathrm{~Hz}\right.$, carbene). ${ }^{31} \mathrm{P}\left\{{ }^{1} \mathrm{H}\right\}$-NMR $\left(T=298 \mathrm{~K}, \mathrm{CDCl}_{3}, \mathrm{ppm}\right) \delta: 25.9,25.8$.

IR (KBr): $\nu_{\mathrm{CO}}=1709,1668 \mathrm{~cm}^{-1}, \nu_{\mathrm{BF}}=1059 \mathrm{~cm}^{-1}$.

Anal. calcd for $\mathrm{C}_{30} \mathrm{H}_{32} \mathrm{BF}_{4} \mathrm{~N}_{4} \mathrm{O}_{2}$ PPd: C 51.12, H 4.58, N 7.95. Found: C 51.42, H 4.32, N 8.10.

\section{Synthesis of complex $4 \mathrm{~b}$}

Complex $\mathbf{4 b}$ was prepared in an analogous manner to that described for 4a starting from $0.0144 \mathrm{~g}$ of $\left[\operatorname{Pd}(\mu-\mathrm{Cl})\left(\eta^{3} \text {-allyl }\right)\right]_{2}$, $0.0358 \mathrm{~g}$ of $3 \mathbf{b} / \mathrm{AgBF}_{4}$ and $0.0196 \mathrm{~g}$ of $\mathrm{PPh}_{3}$.

$0.0511 \mathrm{~g}$ (yield $88 \%$ ) of $\mathbf{4 b}$ was obtained.

${ }^{1} \mathrm{H}-\mathrm{NMR}\left(400 \mathrm{MHz}, T=298 \mathrm{~K}, \mathrm{CDCl}_{3}, \mathrm{ppm}\right) \delta: 2.38(\mathrm{~m}, 1 \mathrm{H}$, anti allyl- $\mathrm{H}$ trans $\mathrm{P}), 2.93(\mathrm{~d}, 1 \mathrm{H}, J=13.6 \mathrm{~Hz}$, anti allyl- $\mathrm{H}$ trans C), $3.17(\mathrm{~d}, 1 \mathrm{H}, J=13.4 \mathrm{~Hz}$, anti allyl-H trans $\mathrm{C}), 3.40(\mathrm{~s}, 3 \mathrm{H}$, $\left.\mathrm{NCH}_{3}\right), 3.42\left(\mathrm{~s}, 3 \mathrm{H}, \mathrm{NCH}_{3}\right), 3.60(\mathrm{~m}, 1 \mathrm{H}$, anti allyl-H trans $\mathrm{P})$, $3.62\left(\mathrm{~s}, 3 \mathrm{H}, \mathrm{NCH}_{3}\right), 3.63\left(\mathrm{~s}, 3 \mathrm{H}, \mathrm{NCH}_{3}\right), 3.86\left(\mathrm{~s}, 3 \mathrm{H}, \mathrm{NCH}_{3}\right)$, $3.96\left(\mathrm{dd}, 1 \mathrm{H}, J_{\mathrm{H}-\mathrm{H}}=6.8 \mathrm{~Hz}, J_{\mathrm{H}-\mathrm{P}}=6.8 \mathrm{~Hz}\right.$, syn allyl-H trans $\left.\mathrm{P}\right)$, $4.09(\mathrm{~d}, 1 \mathrm{H}, J=6.4 \mathrm{~Hz}$, syn allyl-H trans $\mathrm{C}), 4.19(\mathrm{~d}, 1 \mathrm{H}, J=$ $6.1 \mathrm{~Hz}$, syn allyl-H trans $\mathrm{C}), 4.70\left(\mathrm{dd}, 1 \mathrm{H}, J_{\mathrm{H}-\mathrm{H}}=7.7 \mathrm{~Hz}, J_{\mathrm{H}-\mathrm{P}}=\right.$ $7.7 \mathrm{~Hz}$, syn allyl-H trans $\mathrm{P}$ ), 4.80 and 5.57 (AB system, $2 \mathrm{H}, J=$ $\left.14.8 \mathrm{~Hz}, \mathrm{NCH}_{2}\right), 5.03$ and $5.63(\mathrm{AB}$ system, $2 \mathrm{H}, J=15.0 \mathrm{~Hz}$, $\left.\mathrm{NCH}_{2}\right), 5.22(\mathrm{~m}, 1 \mathrm{H}$, central allyl-H), 5.97 (m, 1H, central allyl-H), 7.00-7.60 (m, 40H, 8Ph).

${ }^{13} \mathrm{C}\left\{{ }^{1} \mathrm{H}\right\}$-NMR $\left(T=298 \mathrm{~K}, \mathrm{CDCl}_{3}, \mathrm{ppm}\right) \delta: 28.7\left(\mathrm{CH}_{3}, \mathrm{NCH}_{3}\right)$, $28.8\left(\mathrm{CH}_{3}, \mathrm{NCH}_{3}\right), 31.9\left(\mathrm{CH}_{3}, \mathrm{NCH}_{3}\right), 39.3\left(\mathrm{CH}_{3}, \mathrm{NCH}_{3}\right), 39.5$ $\left(\mathrm{CH}_{3}, \mathrm{NCH}_{3}\right), 52.4\left(\mathrm{CH}_{2}, \mathrm{NCH}_{2}\right), 52.7\left(\mathrm{CH}_{2}, \mathrm{NCH}_{2}\right), 68.6$ (d, $\mathrm{CH}_{2}, J_{\mathrm{C}-\mathrm{P}}=1.9 \mathrm{~Hz}$, allyl trans-C), $68.7\left(\mathrm{~d}, \mathrm{CH}_{2}, J_{\mathrm{C}-\mathrm{P}}=1.8 \mathrm{~Hz}\right.$, allyl trans-C), 70.1 (d, $\mathrm{CH}_{2}, J_{\mathrm{C}-\mathrm{P}}=27.7 \mathrm{~Hz}$, allyl trans-P), 70.8 (d, $\mathrm{CH}_{2}, J_{\mathrm{C}-\mathrm{P}}=27.8 \mathrm{~Hz}$, allyl trans-P), $110.1\left(\mathrm{C}, \mathrm{C}^{5}\right), 110.3\left(\mathrm{C}, \mathrm{C}^{5}\right)$, $121.0(\mathrm{CH}$, central allyl), $122.8(\mathrm{CH}$, central allyl), 127.2-135.3 $(\mathrm{Ph}), 141.1\left(\mathrm{C}^{4}\right), 141.3\left(\mathrm{C}^{4}\right), 143.7(\mathrm{Ph}), 150.3(\mathrm{C}, \mathrm{C}=\mathrm{O}), 150.4$ $(\mathrm{C}, \mathrm{C}=\mathrm{O}), 152.8(\mathrm{C}, \mathrm{C}=\mathrm{O}), 152.9(\mathrm{C}, \mathrm{C}=\mathrm{O}), 187.5\left(\mathrm{~d}, \mathrm{C}, J_{\mathrm{C}-\mathrm{P}}=\right.$ $18.5 \mathrm{~Hz}$, carbene), 187.8 (d, C, $J_{\mathrm{C}-\mathrm{P}}=18.4 \mathrm{~Hz}$, carbene). ${ }^{31} \mathrm{P}\left\{{ }^{1} \mathrm{H}\right\}$-NMR $\left(T=298 \mathrm{~K}, \mathrm{CDCl}_{3}, \mathrm{ppm}\right) \delta: 25.6,26.3$.

IR (KBr): $\nu_{\mathrm{CO}}=1709,1668 \mathrm{~cm}^{-1}, \nu_{\mathrm{BF}}=1056 \mathrm{~cm}^{-1}$.

Anal. calcd for $\mathrm{C}_{36} \mathrm{H}_{36} \mathrm{BF}_{4} \mathrm{~N}_{4} \mathrm{O}_{2}$ PPd: C 55.37, $\mathrm{H}$ 4.65, N 7.17. Found: C 55.12, H 4.80, N 7.38.

\section{Synthesis of complex $4 \mathrm{c}$}

Complex 4c was prepared in an analogous manner to that described for 4a starting from $0.0160 \mathrm{~g}$ of $\left[\mathrm{Pd}(\mu-\mathrm{Cl})\left(\eta^{3} \text {-allyl }\right)\right]_{2}$, $0.0418 \mathrm{~g}$ of $3 \mathrm{c} / \mathrm{AgBF}_{4}$ and $0.0218 \mathrm{~g}$ of $\mathrm{PPh}_{3}$.

$0.0559 \mathrm{~g}$ (yield $84 \%$ ) of $\mathbf{4 c}$ was obtained.

${ }^{1} \mathrm{H}-\mathrm{NMR}\left(400 \mathrm{MHz}, T=298 \mathrm{~K}, \mathrm{CDCl}_{3}, \mathrm{ppm}\right) \delta: 2.97(\mathrm{~d}, 1 \mathrm{H}$, $J=13.3 \mathrm{~Hz}$, anti allyl- $\mathrm{H}$ trans $\mathrm{C}), 3.20(\mathrm{~d}, 1 \mathrm{H}, J=12.8 \mathrm{~Hz}$, anti allyl- $\mathrm{H}$ trans $\mathrm{C}), 3.36(\mathrm{~m}, 1 \mathrm{H}$, anti allyl- $\mathrm{H}$ trans $\mathrm{P}), 3.42(\mathrm{~s}, 6 \mathrm{H}$, $\left.2 \mathrm{NCH}_{3}\right), 3.61\left(\mathrm{~s}, 3 \mathrm{H}, \mathrm{NCH}_{3}\right), 3.62\left(\mathrm{~s}, 3 \mathrm{H}, \mathrm{NCH}_{3}\right), 3.68(\mathrm{~s}, 3 \mathrm{H}$, $\left.\mathrm{NCH}_{3}\right), 3.76(\mathrm{~m}, 1 \mathrm{H}$, anti allyl-H trans $\mathrm{P}), 3.82\left(\mathrm{~s}, 3 \mathrm{H}, \mathrm{NCH}_{3}\right)$, $4.04(\mathrm{~d}, 1 \mathrm{H}, J=6.1 \mathrm{~Hz}$, syn allyl-H trans $\mathrm{C}), 4.22(\mathrm{~d}, 1 \mathrm{H}, J=$ $6.4 \mathrm{~Hz}$, syn allyl-H trans $\mathrm{C}), 4.71\left(\mathrm{dd}, 1 \mathrm{H}, J_{\mathrm{H}-\mathrm{H}}=6.0 \mathrm{~Hz}, J_{\mathrm{H}-\mathrm{P}}=\right.$ $6.8 \mathrm{~Hz}$, syn allyl- $\mathrm{H}$ trans $\mathrm{P}$ ), 4.82 and 5.28 (AB system, $2 \mathrm{H}, J=$ $17.2 \mathrm{~Hz}, \mathrm{NCH}_{2}$ ), 4.88 and $5.47(\mathrm{AB}$ system, $2 \mathrm{H}, J=17.6 \mathrm{~Hz}$, $\left.\mathrm{NCH}_{2}\right), 4.89(\mathrm{~m}, 1 \mathrm{H}$, syn allyl-H trans $\mathrm{P}), 5.63(\mathrm{~m}, 1 \mathrm{H}$, central allyl-H), 6.03 (m, 2H, central allyl-H), 7.15-7.46 (m, 40H, 8Ph). ${ }^{13} \mathrm{C}\left\{{ }^{1} \mathrm{H}\right\}$-NMR $\left(T=298 \mathrm{~K}, \mathrm{CDCl}_{3}, \mathrm{ppm}\right) \delta: 28.7\left(\mathrm{CH}_{3}, \mathrm{NCH}_{3}\right)$, $31.8\left(\mathrm{CH}_{3}, \mathrm{NCH}_{3}\right), 39.4\left(\mathrm{CH}_{3}, \mathrm{NCH}_{3}\right), 40.0\left(\mathrm{CH}_{2}, \mathrm{NCH}_{2}\right), 68.9$ $\left(\mathrm{CH}_{2}\right.$, allyl trans-C), $69.3\left(\mathrm{CH}_{2}\right.$, allyl trans-C), $69.9\left(\mathrm{~d}, \mathrm{CH}_{2}\right.$, $J_{\mathrm{C}-\mathrm{P}}=27.5 \mathrm{~Hz}$, allyl trans $\left.-\mathrm{P}\right), 70.4\left(\mathrm{~d}, \mathrm{CH}_{2}, J_{\mathrm{C}-\mathrm{P}}=27.6 \mathrm{~Hz}\right.$, allyl trans-P), $81.6\left(\mathrm{C}, \equiv \underline{\mathrm{C}}-\mathrm{CH}_{2}\right), 81.9 \quad\left(\mathrm{C}, \equiv \underline{\mathrm{C}}-\mathrm{CH}_{2}\right), 86.5$ (C, $\equiv \underline{\mathrm{C}}-\mathrm{Ph}), 87.4(\mathrm{C}, \equiv \mathrm{C}-\mathrm{Ph}), 109.6\left(\mathrm{C}, \mathrm{C}^{5}\right), 109.7\left(\mathrm{C}, \mathrm{C}^{5}\right), 121.4$ $\left(\mathrm{d}, \mathrm{CH}, J_{\mathrm{C}-\mathrm{P}}=5.1 \mathrm{~Hz}\right.$, central allyl), $122.7\left(\mathrm{~d}, \mathrm{CH}, J_{\mathrm{C}-\mathrm{P}}=5.2 \mathrm{~Hz}\right.$, central allyl), 128.4-134.0 (Ph), 141.2 (C, $\left.\mathrm{C}^{4}\right), 150.4(\mathrm{C}, \mathrm{C}=\mathrm{O})$, $152.6(\mathrm{C}, \mathrm{C}=\mathrm{O}), 152.7(\mathrm{C}, \mathrm{C}=\mathrm{O}), 187.3\left(\mathrm{~d}, \mathrm{C}, J_{\mathrm{C}-\mathrm{P}}=18.8 \mathrm{~Hz}\right.$, carbene), 187.7 (d, C, $J_{\mathrm{C}-\mathrm{P}}=18.5 \mathrm{~Hz}$, carbene). ${ }^{31} \mathrm{P}\left\{{ }^{1} \mathrm{H}\right\}-\mathrm{NMR}$ $\left(T=298 \mathrm{~K}, \mathrm{CDCl}_{3}, \mathrm{ppm}\right) \delta: 25.4,25.9$.

IR (KBr): $\nu_{\mathrm{CO}}=1709,1667 \mathrm{~cm}^{-1}, \nu_{\mathrm{BF}}=1058 \mathrm{~cm}^{-1}$.

Anal. calcd for $\mathrm{C}_{38} \mathrm{H}_{36} \mathrm{BF}_{4} \mathrm{~N}_{4} \mathrm{O}_{2}$ PPd: C 56.70, $\mathrm{H}$ 4.51, N 6.96. Found: C 56.52, H 4.78, N 7.12.

\section{Synthesis of complex 4d}

Complex 4d was prepared in an analogous manner to that described for 4a starting from $0.0154 \mathrm{~g}$ of $\left[\mathrm{Pd}(\mu-\mathrm{Cl})\left(\eta^{3} \text {-allyl }\right)\right]_{2}$, $0.0383 \mathrm{~g}$ of $3 \mathrm{~d} / \mathrm{AgBF}_{4}$ and $0.0210 \mathrm{~g}$ of $\mathrm{PPh}_{3}$.

$0.0522 \mathrm{~g}$ (yield $84 \%$ ) of $4 \mathrm{~d}$ was obtained.

${ }^{1} \mathrm{H}-\mathrm{NMR}\left(400 \mathrm{MHz}, T=298 \mathrm{~K}, \mathrm{CDCl}_{3}, \mathrm{ppm}\right) \delta: 3.13(\mathrm{~d}, 1 \mathrm{H}$, $J=13.4 \mathrm{~Hz}$, anti allyl- $\mathrm{H}$ trans $\mathrm{C}), 3.28(\mathrm{~d}+\mathrm{m}, 2 \mathrm{H}, J=13.2 \mathrm{~Hz}$, anti allyl- $\mathrm{H}$ trans $\mathrm{C}), 3.29(\mathrm{~m}, 1 \mathrm{H}$, anti allyl- $\mathrm{H}$ trans $\mathrm{P}), 3.58(\mathrm{~s}$, $\left.3 \mathrm{H}, \mathrm{NCH}_{3}\right), 3.59\left(\mathrm{~s}, 3 \mathrm{H}, \mathrm{NCH}_{3}\right), 3.60\left(\mathrm{~s}, 3 \mathrm{H}, \mathrm{NCH}_{3}\right), 3.62(\mathrm{~s}, 3 \mathrm{H}$, $\left.\mathrm{NCH}_{3}\right), 3.68(\mathrm{~m}, 1 \mathrm{H}$, anti allyl-H trans $\mathrm{P}), 3.74\left(\mathrm{~s}, 3 \mathrm{H}, \mathrm{NCH}_{3}\right)$, $3.81\left(\mathrm{~s}, 3 \mathrm{H}, \mathrm{NCH}_{3}\right), 4.13(\mathrm{~d}, 1 \mathrm{H}, J=7.4 \mathrm{~Hz}$, syn allyl-H trans $\mathrm{C})$, $4.21(\mathrm{~d}, 1 \mathrm{H}, J=7.8 \mathrm{~Hz}$, syn allyl- $\mathrm{H}$ trans $\mathrm{C}), 4.58\left(\mathrm{dd}, 1 \mathrm{H}, J_{\mathrm{H}-\mathrm{H}}=\right.$ $J_{\mathrm{H}-\mathrm{P}}=5.2 \mathrm{~Hz}$, syn allyl-H trans $\left.\mathrm{P}\right), 4.79\left(\mathrm{dd}, 1 \mathrm{H}, J_{\mathrm{H}-\mathrm{H}}=5.5 \mathrm{~Hz}\right.$, $J_{\mathrm{H}-\mathrm{P}}=5.5 \mathrm{~Hz}$, syn allyl-H trans $\left.\mathrm{P}\right), 5.15\left(\mathrm{~s}, 4 \mathrm{H}, 2 \mathrm{NCH}_{2}\right), 5.70$ (m, 1H, central allyl-H), 5.99 (m, 1H, central allyl-H), 7.25-7.47 $(\mathrm{m}, 40 \mathrm{H}, 8 \mathrm{Ph}) .{ }^{13} \mathrm{C}\left\{{ }^{1} \mathrm{H}\right\}-\mathrm{NMR}\left(T=298 \mathrm{~K}, \mathrm{CDCl}_{3}, \mathrm{ppm}\right) \delta: 31.8$ $\left(\mathrm{CH}_{3}, \mathrm{NCH}_{3}\right), 36.9\left(\mathrm{CH}_{3}, \mathrm{NCH}_{3}\right), 37.0\left(\mathrm{CH}_{3}, \mathrm{NCH}_{3}\right), 38.7\left(\mathrm{CH}_{3}\right.$, $\left.\mathrm{NCH}_{3}\right), 38.9\left(\mathrm{CH}_{3}, \mathrm{NCH}_{3}\right), 45.1\left(\mathrm{CH}_{2}, \mathrm{NCH}_{2}\right), 68.3\left(\mathrm{~d}, \mathrm{CH}_{2}\right.$, $J_{\mathrm{C}-\mathrm{P}}=28.4 \mathrm{~Hz}$, allyl trans-P), $69.1\left(\mathrm{~d}, \mathrm{CH}_{2}, J_{\mathrm{C}-\mathrm{P}}=28.1 \mathrm{~Hz}\right.$, allyl trans-P), $69.2\left(\mathrm{~d}, \mathrm{CH}_{2}, J_{\mathrm{C}-\mathrm{P}}=1.7 \mathrm{~Hz}\right.$, allyl trans-C), $69.4\left(\mathrm{~d}, \mathrm{CH}_{2}\right.$, $J_{\mathrm{C}-\mathrm{P}}=1.9 \mathrm{~Hz}$, allyl trans-C), $110.7\left(\mathrm{C}, \mathrm{C}^{5}\right), 121.3\left(\mathrm{~d}, \mathrm{CH}, J_{\mathrm{C}-\mathrm{P}}=\right.$ $5.5 \mathrm{~Hz}$, central allyl), 122.8 (d, CH, $J_{\mathrm{C}-\mathrm{P}}=5.3 \mathrm{~Hz}$, central allyl), 127.9-136.4 (Ph), 141.0 (C, $\left.\mathrm{C}^{4}\right), 141.1\left(\mathrm{C}, \mathrm{C}^{4}\right), 150.2(\mathrm{C}, \mathrm{C}=\mathrm{O})$, $152.7(\mathrm{C}, \mathrm{C}=\mathrm{O}), 186.1$ (d, C, $J_{\mathrm{C}-\mathrm{P}}=19.3 \mathrm{~Hz}$, carbene), 186.5 (d, 
$\mathrm{C}, J_{\mathrm{C}-\mathrm{P}}=19.4 \mathrm{~Hz}$, carbene). ${ }^{31} \mathrm{P}\left\{{ }^{1} \mathrm{H}\right\}-\mathrm{NMR}\left(T=298 \mathrm{~K}, \mathrm{CDCl}_{3}\right.$, ppm) $\delta: 25.9,26.0$.

IR (KBr): $\nu_{\mathrm{CO}}=1707,1668 \mathrm{~cm}^{-1}, \nu_{\mathrm{BF}}=1056 \mathrm{~cm}^{-1}$.

Anal. calcd for $\mathrm{C}_{36} \mathrm{H}_{36} \mathrm{BF}_{4} \mathrm{~N}_{4} \mathrm{O}_{2} \mathrm{PPd}$ : C 55.37, $\mathrm{H}$ 4.65, N 7.17. Found: C 55.22, H 4.58, N 7.42.

\section{Synthesis of complex $5 \mathrm{a}$}

$0.0411 \mathrm{~g}(0.112 \mathrm{mmol})$ of the dimer $\left[\mathrm{Pd}(\mu-\mathrm{Cl})\left(\eta^{3}-\mathrm{C}_{3} \mathrm{H}_{5}\right)\right]_{2}$ and $0.0353 \mathrm{~g}$ (0.224 mmol) of PTA (1,3,5-triaza-7-phosphadamantane) were dissolved in ca. $30 \mathrm{~mL}$ of anhydrous $\mathrm{CH}_{3} \mathrm{CN}$ in a $100 \mathrm{~mL}$ two necked flask under an inert atmosphere (Ar). The resulting mixture was treated with $0.0787 \mathrm{~g}(0.0975 \mathrm{mmol})$ of $3 \mathrm{a} / \mathrm{AgBF}_{4}$ and stirred at RT for ca. 1 hour.

The precipitated $\mathrm{AgCl}$ was removed by filtration on a Millipore membrane filter.

The solution was dried under vacuum and the residue was treated with $2 \mathrm{~mL}$ of $\mathrm{CH}_{2} \mathrm{Cl}_{2}$.

Addition of diethyl ether to the concentrated solution yielded the precipitation of complex 5a as a brownish solid, which was filtered off on a Gooch, washed with $n$-pentane and dried under vacuum.

$0.0991 \mathrm{~g}$ of 5 a was obtained (yield 97\%).

${ }^{1} \mathrm{H}-\mathrm{NMR}\left(400 \mathrm{MHz}, T=298 \mathrm{~K}, \mathrm{D}_{2} \mathrm{O}, \mathrm{ppm}\right) \delta: 2.88(\mathrm{~d}, 2 \mathrm{H}, J=$ 13.2 Hz, 2 anti allyl-H trans-C), 3.15 (m, 2H, 2 anti allyl-H trans-P), $3.32\left(\mathrm{~s}, 3 \mathrm{H}, \mathrm{NCH}_{3}\right), 3.33\left(\mathrm{~s}, 3 \mathrm{H}, \mathrm{NCH}_{3}\right), 3.74\left(\mathrm{~s}, 3 \mathrm{H}, \mathrm{NCH}_{3}\right)$, $3.77\left(\mathrm{~s}, 3 \mathrm{H}, \mathrm{NCH}_{3}\right), 3.80\left(\mathrm{~s}, 3 \mathrm{H}, \mathrm{NCH}_{3}\right), 3.87$ (s, 3H, $\mathrm{NCH}_{3}$ ), $3.92\left(\mathrm{~s}, 3 \mathrm{H}, \mathrm{NCH}_{3}\right), 4.05\left(\mathrm{~s}, 3 \mathrm{H}, \mathrm{NCH}_{3}\right), 4.21(\mathrm{~s}, 12 \mathrm{H}$, $6 \mathrm{NCH}_{2} \mathrm{P}_{\mathrm{PTA}}$ ), 4.38 (d, 2H, $J=7.4 \mathrm{~Hz}, 2$ syn allyl-H trans-C), 4.47 (m, 2H, 2 syn allyl-H trans-P), $4.55\left(\mathrm{~m}, 12 \mathrm{H}, 6 \mathrm{NCH}_{2} \mathrm{~N}_{\text {PTA }}\right), 5.48$ (m, 2H, 2 central allyl-H). ${ }^{13} \mathrm{C}\left\{{ }^{1} \mathrm{H}\right\}-\mathrm{NMR}\left(T=298 \mathrm{~K}, \mathrm{D}_{2} \mathrm{O}, \mathrm{ppm}\right)$ $\delta$ : $28.4\left(\mathrm{CH}_{3}, \mathrm{NCH}_{3}\right), 31.8\left(\mathrm{CH}_{3}, \mathrm{NCH}_{3}\right), 36.7\left(\mathrm{CH}_{3}, \mathrm{NCH}_{3}\right), 36.9$ $\left(\mathrm{CH}_{3}, \mathrm{NCH}_{3}\right), 38.2\left(\mathrm{CH}_{3}, \mathrm{NCH}_{3}\right), 38.4\left(\mathrm{CH}_{3}, \mathrm{NCH}_{3}\right), 50.3\left(\mathrm{CH}_{2}\right.$, $\left.\mathrm{NCH}_{2} \mathrm{P}, J_{\mathrm{C}-\mathrm{P}}=13.6 \mathrm{~Hz}\right), 62.7\left(\mathrm{CH}_{2}\right.$, allyl trans-C $), 62.8\left(\mathrm{CH}_{2}\right.$, allyl trans-C), 68.9 (d, $\mathrm{CH}_{2}, J_{\mathrm{C}-\mathrm{P}}=4.8 \mathrm{~Hz}$, allyl trans-P), 69.2 (d, $\mathrm{CH}_{2}, J_{\mathrm{C}-\mathrm{P}}=5.2 \mathrm{~Hz}$, allyl trans-P), $70.7\left(\mathrm{CH}_{2}, \mathrm{NCH}_{2} \mathrm{~N}\right), 70.8$ $\left(\mathrm{CH}_{2}, \mathrm{NCH}_{2} \mathrm{~N}\right), 111.2\left(\mathrm{C}, \mathrm{C}^{5}\right), 111.4\left(\mathrm{C}, \mathrm{C}^{5}\right), 121.9(\mathrm{CH}$, central allyl), 122.0 ( $\mathrm{CH}$, central allyl), $141.8\left(\mathrm{C}, \mathrm{C}^{4}\right), 141.9\left(\mathrm{C}, \mathrm{C}^{4}\right)$, $151.8(\mathrm{C}, \mathrm{C}=\mathrm{O}), 154.5(\mathrm{C}, \mathrm{C}=\mathrm{O}), 183.9\left(\mathrm{C}, J_{\mathrm{C}-\mathrm{P}}=21.2 \mathrm{~Hz}\right.$, carbene). ${ }^{31} \mathrm{P}\left\{{ }^{1} \mathrm{H}\right\}$-NMR $\left(T=298 \mathrm{~K}, \mathrm{D}_{2} \mathrm{O}, \mathrm{ppm}\right) \delta$ : -52.4 .

IR (KBr): $\nu_{\mathrm{CO}}=1704,1665 \mathrm{~cm}^{-1}, \nu_{\mathrm{BF}}=1031,1084 \mathrm{~cm}^{-1}$.

Anal. calcd for $\mathrm{C}_{18} \mathrm{H}_{29} \mathrm{BF}_{4} \mathrm{~N}_{7} \mathrm{O}_{2} \mathrm{PPd}: \mathrm{C}$ 36.05, $\mathrm{H}$ 4.87, $\mathrm{N}$ 16.35. Found: C 36.34, H 4.97, N 16.14.

\section{Synthesis of complex $5 \mathrm{~b}$}

Complex $5 \mathbf{b}$ was prepared in an analogous manner to that described for 5a starting from $0.0353 \mathrm{~g}$ of $\left[\mathrm{Pd}(\mu-\mathrm{Cl})\left(\eta^{3} \text {-allyl }\right)\right]_{2}$, $0.0804 \mathrm{~g}$ of $3 \mathrm{~b} / \mathrm{AgBF}_{4}$ and $0.0304 \mathrm{~g}$ of PTA.

$0.0925 \mathrm{~g}$ (yield $82 \%$ ) of $\mathbf{5 b}$ was obtained.

${ }^{1} \mathrm{H}-\mathrm{NMR}\left(400 \mathrm{MHz}, T=298 \mathrm{~K}, \mathrm{D}_{2} \mathrm{O}, \mathrm{ppm}\right) \delta: 2.76(\mathrm{~m}, 2 \mathrm{H}$, 2 anti allyl-H trans-C), $3.06(\mathrm{~m}, 2 \mathrm{H}, 2$ anti allyl-H trans-P), 3.28 (s, $\left.3 \mathrm{H}, \mathrm{NCH}_{3}\right), 3.29\left(\mathrm{~s}, 3 \mathrm{H}, \mathrm{NCH}_{3}\right), 3.72\left(\mathrm{~s}, 6 \mathrm{H}, 3 \mathrm{NCH}_{2} \mathrm{P}_{\mathrm{PTA}}\right)$, $3.75\left(\mathrm{~s}, 6 \mathrm{H}, 3 \mathrm{NCH}_{2} \mathrm{P}_{\mathrm{PTA}}\right), 3.77\left(\mathrm{~s}, 3 \mathrm{H}, \mathrm{NCH}_{3}\right), 3.80(\mathrm{~s}, 3 \mathrm{H}$, $\left.\mathrm{NCH}_{3}\right), 3.88\left(\mathrm{~s}, 3 \mathrm{H}, \mathrm{NCH}_{3}\right), 4.06\left(\mathrm{~s}, 3 \mathrm{H}, \mathrm{NCH}_{3}\right), 4.27-4.70(\mathrm{~m}$, $16 \mathrm{H}, 6 \mathrm{NCH}_{2} \mathrm{~N}_{\mathrm{PTA}}$ and 2 syn allyl-H trans-C and 2 syn allyl-H trans-P), 5.07 and 5.64 (AB system, $2 \mathrm{H}, J=15.4 \mathrm{~Hz}, \mathrm{NCH}_{2}$ ), 5.29 and $5.76\left(\mathrm{AB}\right.$ system, $\left.2 \mathrm{H}, J=15.7 \mathrm{~Hz}, \mathrm{NCH}_{2}\right), 5.36(\mathrm{~m}, 2 \mathrm{H}$,
2 central allyl-H), 6.81-7.36 (m, 10H, 2Ph). ${ }^{13} \mathrm{C}\left\{{ }^{1} \mathrm{H}\right\}-\mathrm{NMR}(T=$ $\left.298 \mathrm{~K}, \mathrm{D}_{2} \mathrm{O}, \mathrm{ppm}\right) \delta: 28.5\left(\mathrm{CH}_{3}, \mathrm{NCH}_{3}\right), 31.9\left(\mathrm{CH}_{3}, \mathrm{NCH}_{3}\right), 38.6$ $\left(\mathrm{CH}_{3}, \mathrm{NCH}_{3}\right), 38.9\left(\mathrm{CH}_{3}, \mathrm{NCH}_{3}\right), 49.9$ (d, $\mathrm{CH}_{2}, J=13.5 \mathrm{~Hz}$, $\mathrm{NCH}_{2} \mathrm{P}$ ), 50.0 (d, $\left.\mathrm{CH}_{2}, J=13.6 \mathrm{~Hz}, \mathrm{NCH}_{2} \mathrm{P}\right), 52.5\left(\mathrm{CH}_{2}, \mathrm{NCH}_{2}\right)$, $52.7\left(\mathrm{CH}_{2}, \mathrm{NCH}_{2}\right), 62.6\left(\mathrm{CH}_{2}\right.$, allyl trans-C), $63.0\left(\mathrm{CH}_{2}\right.$, allyl trans-C), 69.4 (d, $\mathrm{CH}_{2}, J_{\mathrm{C}-\mathrm{P}}=26.7 \mathrm{~Hz}$, allyl trans-P), 69.9 (d, $\mathrm{CH}_{2}, J_{\mathrm{C}-\mathrm{P}}=27.8 \mathrm{~Hz}$, allyl trans-P), $70.4\left(\mathrm{CH}_{2}, \mathrm{NCH}_{2} \mathrm{~N}_{\mathrm{PTA}}\right) 70.5$ $\left(\mathrm{CH}_{2}, \mathrm{NCH}_{2} \mathrm{~N}\right), 111.1\left(\mathrm{C}, \mathrm{C}^{5}\right), 111.4\left(\mathrm{C}, \mathrm{C}^{5}\right), 121.9(\mathrm{CH}$, central allyl), 126.9-136.7 (Ph), $141.8\left(\mathrm{C}, \mathrm{C}^{4}\right), 141.9\left(\mathrm{C}, \mathrm{C}^{4}\right), 151.8$ (C, $\mathrm{C}=\mathrm{O}), 154.2(\mathrm{C}, \mathrm{C}=\mathrm{O}), 186.0\left(\mathrm{~d}, \mathrm{C}, J_{\mathrm{C}-\mathrm{P}}=20.4\right.$, carbene $), 186.1$ (d, C, $J_{\mathrm{C}-\mathrm{P}}=20.5 \mathrm{~Hz}$, carbene). ${ }^{31} \mathrm{P}\left\{{ }^{1} \mathrm{H}\right\}$-NMR $\left(T=298 \mathrm{~K}, \mathrm{D}_{2} \mathrm{O}\right.$, ppm) $\delta:-54.1,-54.0$.

IR (KBr): $\nu_{\mathrm{CO}}=1705,1664 \mathrm{~cm}^{-1}, \nu_{\mathrm{BF}}=1035,1083 \mathrm{~cm}^{-1}$.

Anal. calcd for $\mathrm{C}_{24} \mathrm{H}_{33} \mathrm{BF}_{4} \mathrm{~N}_{7} \mathrm{O}_{2} \mathrm{PPd}$ : $\mathrm{C}$ 42.66, $\mathrm{H}$ 4.92, $\mathrm{N}$ 14.51. Found: C 42.82, H 4.78, N 14.22.

\section{Synthesis of complex $5 d$}

Complex 5d was prepared in an analogous manner to that described for 5a starting from $0.0352 \mathrm{~g}$ of $\left[\mathrm{Pd}(\mu-\mathrm{Cl})\left(\eta^{3} \text {-allyl }\right)\right]_{2}$, $0.0800 \mathrm{~g}$ of $3 \mathrm{~d} / \mathrm{AgBF}_{4}$ and $0.0301 \mathrm{~g}$ of PTA.

$0.1057 \mathrm{~g}$ (yield $94 \%$ ) of $\mathbf{5 d}$ was obtained.

${ }^{1} \mathrm{H}-\mathrm{NMR}\left(400 \mathrm{MHz}, T=298 \mathrm{~K}, \mathrm{D}_{2} \mathrm{O}, \mathrm{ppm}\right) \delta: 2.83(\mathrm{~d}, J=$ $13.8 \mathrm{~Hz}, 2 \mathrm{H}, 2$ anti allyl-H trans-C), 3.07 (m, 2H, 2 anti allyl- $\mathrm{H}$ trans-P), $3.69\left(\mathrm{~s}, 3 \mathrm{H}, \mathrm{NCH}_{3}\right), 3.70\left(\mathrm{~s}, 3 \mathrm{H}, \mathrm{NCH}_{3}\right), 3.73(\mathrm{~s}, 3 \mathrm{H}$, $\left.\mathrm{NCH}_{3}\right), 3.82\left(\mathrm{~s}, 3 \mathrm{H}, \mathrm{NCH}_{3}\right), 3.87\left(\mathrm{~s}, 3 \mathrm{H}, \mathrm{NCH}_{3}\right), 4.01(\mathrm{~s}, 3 \mathrm{H}$, $\mathrm{NCH}_{3}$ ), 4.15 (s, 6H, $3 \mathrm{NCH}_{2} \mathrm{P}_{\text {PTA }}$ ), 4.27 (s, 6H, $3 \mathrm{NCH}_{2} \mathrm{P}_{\text {PTA }}$ ), 4.32 (d, $J=6.6 \mathrm{~Hz}, 2 \mathrm{H}, 2$ syn allyl-H trans-C), 4.45 (m, 2H, 2 syn allyl-H trans-P), 4.50-4.70 (m, 12H, $\left.6 \mathrm{NCH}_{2} \mathrm{P}_{\mathrm{PTA}}\right), 5.10\left(2 \mathrm{~s}, 4 \mathrm{H}, 2 \mathrm{~N}-\mathrm{CH}_{2}\right)$, 5.42 (m, 2H, 2 central allyl-H), 7.25-7.30 (m, 10H, 2Ph).

${ }^{13} \mathrm{C}\left\{{ }^{1} \mathrm{H}\right\}$-NMR $\left(T=298 \mathrm{~K}, \mathrm{D}_{2} \mathrm{O}, \mathrm{ppm}\right) \delta: 31.9\left(\mathrm{CH}_{3}, \mathrm{NCH}_{3}\right)$, $36.8\left(\mathrm{CH}_{3}, \mathrm{NCH}_{3}\right), 37.1\left(\mathrm{CH}_{3}, \mathrm{NCH}_{3}\right), 38.2\left(\mathrm{CH}_{3}, \mathrm{NCH}_{3}\right), 38.5$ $\left(\mathrm{CH}_{3}, \mathrm{NCH}_{3}\right), 45.2\left(\mathrm{CH}_{2}, \mathrm{NCH}_{2}\right), 50.3\left(\mathrm{~d}, \mathrm{CH}_{2}, J_{\mathrm{C}-\mathrm{P}}=13.8 \mathrm{~Hz}\right.$, $\mathrm{NCH}_{2} \mathrm{P}$ ), 51.1 (d, $\left.\mathrm{CH}_{2}, J_{\mathrm{C}-\mathrm{P}}=15.4 \mathrm{~Hz}, \mathrm{NCH}_{2} \mathrm{P}\right), 62.8\left(\mathrm{CH}_{2}\right.$, allyl trans-C), $62.9\left(\mathrm{CH}_{2}\right.$, allyl trans-C), $69.2\left(\mathrm{~d}, \mathrm{CH}_{2}, J_{\mathrm{C}-\mathrm{P}}=27.7 \mathrm{~Hz}\right.$, allyl trans-P), $70.8\left(\mathrm{CH}_{2}, \mathrm{NCH}_{2} \mathrm{~N}\right), 71.3\left(\mathrm{~d}, \mathrm{CH}_{2}, J_{\mathrm{C}-\mathrm{P}}=26.2 \mathrm{~Hz}\right.$, allyl trans-P), $111.4\left(\mathrm{C}^{5}\right), 122.0(\mathrm{CH}$, central allyl), $123.0(\mathrm{CH}$, central allyl), 127.2-136.2 (Ph), $142.3\left(\mathrm{C}, \mathrm{C}^{4}\right), 151.6(\mathrm{C}, \mathrm{C}=\mathrm{O})$, $154.2(\mathrm{C}, \mathrm{C}=\mathrm{O}), 184.2$ (d, C, $J_{\mathrm{C}-\mathrm{P}}=19.0 \mathrm{~Hz}$, carbene), 184.3 (d, $\mathrm{C}, J_{\mathrm{C}-\mathrm{P}}=19.8 \mathrm{~Hz}$, carbene). ${ }^{31} \mathrm{P}\left\{{ }^{1} \mathrm{H}\right\}$-NMR $\left(T=298 \mathrm{~K}, \mathrm{D}_{2} \mathrm{O}\right.$, ppm) $\delta:-54.2,-53.1$.

IR (KBr): $\nu_{\mathrm{CO}}=1708,1668 \mathrm{~cm}^{-1}, \nu_{\mathrm{BF}}=1062,1084 \mathrm{~cm}^{-1}$.

Anal. calcd for $\mathrm{C}_{24} \mathrm{H}_{33} \mathrm{BF}_{4} \mathrm{~N}_{7} \mathrm{O}_{2} \mathrm{PPd}$ : $\mathrm{C}$ 42.66, $\mathrm{H}$ 4.92, $\mathrm{N}$ 14.51. Found: C 42.52, H 4.99, N 14.32.

\section{Synthesis of complex 6a}

$0.0173 \mathrm{~g}(0.047 \mathrm{mmol})$ of the dimer $\left[\mathrm{Pd}(\mu-\mathrm{Cl})\left(\eta^{3}-\mathrm{C}_{3} \mathrm{H}_{5}\right)\right]_{2}$ and $0.0124 \mathrm{~g}$ ( $0.094 \mathrm{mmol})$ of DIC (2,6-dimethylphenyl isocyanide) were dissolved in ca. $20 \mathrm{~mL}$ of anhydrous $\mathrm{CH}_{3} \mathrm{CN}$ in a $50 \mathrm{~mL}$ two necked flask under an inert atmosphere (Ar). The resulting mixture was treated with $0.0344 \mathrm{~g}(0.043 \mathrm{mmol})$ of $3 \mathrm{a} / \mathrm{AgBF}_{4}$ and stirred at RT for $c a .15 \mathrm{~min}$.

The precipitated $\mathrm{AgCl}$ was removed by filtration on a Millipore membrane filter.

Addition of diethyl ether to the concentrated solution yielded the precipitation of complex $\mathbf{6 a}$ as a brownish solid, which was filtered off on a Gooch and washed with $n$-pentane. 
$0.0431 \mathrm{~g}$ of $6 \mathrm{a}$ was obtained (yield $88 \%$ ).

${ }^{1} \mathrm{H}-\mathrm{NMR}\left(400 \mathrm{MHz}, T=298 \mathrm{~K}, \mathrm{CDCl}_{3}, \mathrm{ppm}\right) \delta: 2.41(\mathrm{~s}, 6 \mathrm{H}$, $\left.2 \mathrm{CH}_{3}{ }^{\mathrm{DIC}}\right), 3.20(\mathrm{~d}, 1 \mathrm{H}, J=13.3 \mathrm{~Hz}$, anti allyl-H), $3.40(\mathrm{~s}, 3 \mathrm{H}$, $\left.\mathrm{NCH}_{3}\right), 3.41$ (d, $1 \mathrm{H}, J=12.7 \mathrm{~Hz}$, anti allyl-H), $3.88(\mathrm{~s}, 3 \mathrm{H}$, $\left.\mathrm{NCH}_{3}\right), 4.05$ (s, 3H, $\left.\mathrm{NCH}_{3}\right), 4.20\left(\mathrm{~s}, 3 \mathrm{H}, \mathrm{NCH}_{3}\right), 4.47$ (d, $1 \mathrm{H}, J=$ $6.4 \mathrm{~Hz}$, syn allyl-H), 4.79 (d, $1 \mathrm{H}, J=7.5 \mathrm{~Hz}$, syn allyl-H), 5.72 (m, 1H, central allyl-H), 7.17-7.31 (m, 3H, $\left.\mathrm{Ph}^{\mathrm{DIC}}\right) .{ }^{13} \mathrm{C}\left\{{ }^{1} \mathrm{H}\right\}-\mathrm{NMR}$ $\left(T=298 \mathrm{~K}, \mathrm{CDCl}_{3}, \mathrm{ppm}\right) \delta: 18.7\left(\mathrm{CH}_{3}, \mathrm{CH}_{3}{ }^{\mathrm{DIC}}\right), 28.6\left(\mathrm{CH}_{3}\right.$, $\left.\mathrm{NCH}_{3}\right), 32.0\left(\mathrm{CH}_{3}, \mathrm{NCH}_{3}\right), 37.5\left(\mathrm{CH}_{3}, \mathrm{NCH}_{3}\right), 39.3\left(\mathrm{CH}_{3}, \mathrm{NCH}_{3}\right)$, $65.0\left(\mathrm{CH}_{2}\right.$, allyl-C), $69.3\left(\mathrm{CH}_{2}\right.$, allyl-C), $110.8\left(\mathrm{C}, \mathrm{C}^{5}\right), 122.2$ (central allyl), 128.4-135.6 (Ph), $141.4\left(\mathrm{C}, \mathrm{C}^{4}\right), 150.6(\mathrm{C}, \mathrm{C}=\mathrm{O})$, $150.7\left(\mathrm{C}, \mathrm{CN}^{\mathrm{DIC}}\right), 153.4(\mathrm{C}, \mathrm{C}=\mathrm{O}), 181.5$ (C, carbene).

IR $(\mathrm{KBr}): \nu_{\mathrm{CN}}=2175 \mathrm{~cm}^{-1}, \nu_{\mathrm{CO}}=1706,1665 \mathrm{~cm}^{-1}, \nu_{\mathrm{BF}}=$ $1056 \mathrm{~cm}^{-1}$.

Anal. calcd for $\mathrm{C}_{21} \mathrm{H}_{26} \mathrm{BF}_{4} \mathrm{~N}_{5} \mathrm{O}_{2} \mathrm{Pd}$ : $\mathrm{C} 43.97, \mathrm{H}$ 4.57, $\mathrm{N}$ 12.21. Found: C 44.24, H 4.77, N 12.04.

\section{Synthesis of complex $6 \mathrm{~b}$}

Complex 6b was prepared in an analogous manner to that described for 6a starting from $0.0180 \mathrm{~g}$ of $\left[\mathrm{Pd}(\mu-\mathrm{Cl})\left(\eta^{3} \text {-allyl }\right)\right]_{2}$, $0.0428 \mathrm{~g}$ of $3 \mathrm{~b} / \mathrm{AgBF}_{4}$ and $0.128 \mathrm{~g}$ of DIC.

$0.0491 \mathrm{~g}$ (yield $85 \%$ ) of $\mathbf{6 b}$ was obtained.

${ }^{1} \mathrm{H}-\mathrm{NMR}\left(400 \mathrm{MHz}, T=298 \mathrm{~K}, \mathrm{CDCl}_{3}, \mathrm{ppm}\right) \delta: 2.36(\mathrm{~s}, 6 \mathrm{H}$, $2 \mathrm{CH}_{3}{ }^{\mathrm{DIC}}$ ), 3.42 (s, 3H, $\mathrm{NCH}_{3}$ ), 3.48 (bd, 2H, 2anti allyl-H), 3.91 (s, $3 \mathrm{H}, \mathrm{NCH}_{3}$ ), 4.24 (bd, $1 \mathrm{H}$, syn allyl-H), 4.25 (s, 3H, $\mathrm{NCH}_{3}$ ), 4.67 (d, $1 \mathrm{H}, J=7.5 \mathrm{~Hz}$, syn allyl-H), $5.65\left(\mathrm{~s}, 2 \mathrm{H}, \mathrm{NCH}_{2}\right), 5.73(\mathrm{~m}$, $1 \mathrm{H}$, central allyl-H), 7.16-7.33 (m, 8H, $\mathrm{Ph}$ and $\left.\mathrm{Ph}^{\mathrm{DIC}}\right) .{ }^{13} \mathrm{C}\left\{{ }^{1} \mathrm{H}\right\}-$ NMR $\left(T=298 \mathrm{~K}, \mathrm{CDCl}_{3}, \mathrm{ppm}\right) \delta: 18.8\left(\mathrm{CH}_{3}, \mathrm{CH}_{3}{ }^{\mathrm{DIC}}\right), 28.7\left(\mathrm{CH}_{3}\right.$, $\left.\mathrm{NCH}_{3}\right), 32.1\left(\mathrm{CH}_{3}, \mathrm{NCH}_{3}\right), 39.9\left(\mathrm{CH}_{3}, \mathrm{NCH}_{3}\right), 53.0\left(\mathrm{CH}_{2}, \mathrm{NCH}_{2}\right)$, $65.5\left(\mathrm{CH}_{2}\right.$, allyl-C), $110.6\left(\mathrm{C}, \mathrm{C}^{5}\right), 121.9$ (CH, central allyl), 127.5-135.9 (Ph), $141.5\left(\mathrm{C}, \mathrm{C}^{4}\right), 150.5(\mathrm{C}, \mathrm{C}=\mathrm{O}), 150.7$ (C, $\left.\mathrm{CN}^{\mathrm{DIC}}\right), 153.2(\mathrm{C}, \mathrm{C}=\mathrm{O}), 183.5$ (C, carbene).

IR $(\mathrm{KBr}): \nu_{\mathrm{CN}}=2175 \mathrm{~cm}^{-1}, \nu_{\mathrm{CO}}=1709,1670 \mathrm{~cm}^{-1}, \nu_{\mathrm{BF}}=$ $1057 \mathrm{~cm}^{-1}$.

Anal. calcd for $\mathrm{C}_{27} \mathrm{H}_{30} \mathrm{BF}_{4} \mathrm{~N}_{5} \mathrm{O}_{2} \mathrm{Pd}$ : C 49.91, H 4.65, N 10.78. Found: C 49.84, H 4.83, N 10.90.

\section{Synthesis of complex $6 \mathrm{~d}$}

Complex 6d was prepared in an analogous manner to that described for 6a starting from $0.0184 \mathrm{~g}$ of $\left[\mathrm{Pd}(\mu-\mathrm{Cl})\left(\eta^{3} \text {-allyl }\right)\right]_{2}$, $0.0437 \mathrm{~g}$ of $3 \mathrm{~d} / \mathrm{AgBF}_{4}$ and $0.0131 \mathrm{~g}$ of DIC.

$0.0543 \mathrm{~g}$ (yield $92 \%$ ) of $\mathbf{6 d}$ was obtained.

${ }^{1} \mathrm{H}-\mathrm{NMR}\left(400 \mathrm{MHz}, T=298 \mathrm{~K}, \mathrm{CDCl}_{3}, \mathrm{ppm}\right) \delta: 2.41(\mathrm{~s}, 6 \mathrm{H}$, $\left.2 \mathrm{CH}_{3}{ }^{\mathrm{DIC}}\right), 3.19(\mathrm{~d}, 1 \mathrm{H}, J=13.5 \mathrm{~Hz}$, anti allyl-H), $3.41(\mathrm{~d}, 1 \mathrm{H}, J=$ $12.5 \mathrm{~Hz}$, anti allyl-H), $3.86\left(\mathrm{~s}, 3 \mathrm{H}, \mathrm{NCH}_{3}\right), 4.05\left(\mathrm{~s}, 3 \mathrm{H}, \mathrm{NCH}_{3}\right)$, 4.19 (s, 3H, $\left.\mathrm{NCH}_{3}\right), 4.47$ (d, 1H, J = $7.1 \mathrm{~Hz}$, syn allyl-H), 4.78 (d, $1 \mathrm{H}, J=7.5 \mathrm{~Hz}$, syn allyl-H), $5.18\left(\mathrm{~s}, 2 \mathrm{H}, \mathrm{NCH}_{2}\right), 5.72(\mathrm{~m}, 1 \mathrm{H}$, central allyl-H), 7.17-7.49 (m, 8H, $\mathrm{Ph}$ and $\left.\mathrm{Ph}^{\mathrm{DIC}}\right) .{ }^{13} \mathrm{C}\left\{{ }^{1} \mathrm{H}\right\}-\mathrm{NMR}$ $\left(T=298 \mathrm{~K}, \mathrm{CDCl}_{3}, \mathrm{ppm}\right) \delta: 18.8\left(\mathrm{CH}_{3}, \mathrm{CH}_{3}{ }^{\mathrm{DIC}}\right), 32.0\left(\mathrm{CH}_{3}\right.$, $\left.\mathrm{NCH}_{3}\right), 37.5\left(\mathrm{CH}_{3}, \mathrm{NCH}_{3}\right), 39.3\left(\mathrm{CH}_{3}, \mathrm{NCH}_{3}\right), 45.2\left(\mathrm{CH}_{2}, \mathrm{NCH}_{2}\right)$, $64.9\left(\mathrm{CH}_{2}\right.$, allyl-C), $110.9\left(\mathrm{C}, \mathrm{C}^{5}\right), 122.2(\mathrm{CH}$, central allyl), 127.8-136.3 (Ph), $141.5\left(\mathrm{C}, \mathrm{C}^{4}\right), 150.5(\mathrm{C}, \mathrm{C}=\mathrm{O}), 150.6(\mathrm{C}$, $\left.\mathrm{CN}^{\mathrm{DIC}}\right), 153.2(\mathrm{C}, \mathrm{C}=\mathrm{O}), 181.8(\mathrm{C}$, carbene).

IR $(\mathrm{KBr}): \nu_{\mathrm{CN}}=2173 \mathrm{~cm}^{-1}, \nu_{\mathrm{CO}}=1707,1665 \mathrm{~cm}^{-1}, \nu_{\mathrm{BF}}=$ $1056 \mathrm{~cm}^{-1}$.
Anal. calcd for $\mathrm{C}_{27} \mathrm{H}_{30} \mathrm{BF}_{4} \mathrm{~N}_{5} \mathrm{O}_{2} \mathrm{Pd}$ : C 49.91, $\mathrm{H}$ 4.65, N 10.78. Found: C 50.04, H 4.51, N 10.95.

\section{Synthesis of complex $8 a$}

$0.0156 \mathrm{~g}(0.086 \mathrm{mmol})$ of the dimer $\left[\mathrm{Pd}(\mu-\mathrm{Cl})\left(\eta^{3}-\mathrm{C}_{3} \mathrm{H}_{5}\right)\right]_{2}$ was dissolved in ca. $20 \mathrm{~mL}$ of anhydrous $\mathrm{CH}_{3} \mathrm{CN}$ in a $50 \mathrm{~mL}$ two necked flask under an inert atmosphere (Ar).

The resulting mixture was treated with $0.0690 \mathrm{~g}$ $(0.171 \mathrm{mmol})$ of $3 \mathrm{a} / \mathrm{AgBF}_{4}, 0.0142 \mathrm{~g}(0.086 \mathrm{mmol})$ of $\mathrm{KI}$ and stirred at RT for ca. $15 \mathrm{~min}$. The precipitated $\mathrm{AgCl}$ and $\mathrm{AgI}$ were removed by filtration on a Millipore membrane filter. Addition of diethyl ether to the concentrated solution yielded the precipitation of complex $\mathbf{8 a}$ as a white solid, which was filtered off on a Gooch and washed with $n$-pentane.

$0.0446 \mathrm{~g}$ of $8 \mathrm{a}$ was obtained (yield $80 \%$ ).

${ }^{1} \mathrm{H}-\mathrm{NMR}\left(400 \mathrm{MHz}, T=298 \mathrm{~K}, \mathrm{CD}_{3} \mathrm{CN}, \mathrm{ppm}\right) \delta: 2.95(\mathrm{~d}, 2 \mathrm{H}$, $J=13.3 \mathrm{~Hz}$, anti allyl-H), $3.30\left(\mathrm{~s}, 6 \mathrm{H}, \mathrm{NCH}_{3}\right), 3.73(\mathrm{~s}, 6 \mathrm{H}$, $\mathrm{NCH}_{3}$ ), $3.93\left(\mathrm{~s}, 6 \mathrm{H}, \mathrm{NCH}_{3}\right), 4.02\left(\mathrm{~s}, 6 \mathrm{H}, \mathrm{NCH}_{3}\right), 4.12$ (d, 2H, $J=$ $7.4 \mathrm{~Hz}$, syn allyl-H), 5.59 (m, 1H, central allyl-H). ${ }^{13} \mathrm{C}\left\{{ }^{1} \mathrm{H}\right\}-\mathrm{NMR}$ $\left(T=298 \mathrm{~K}, \mathrm{CD}_{3} \mathrm{CN}, \mathrm{ppm}\right) \delta: 28.4\left(\mathrm{CH}_{3}, \mathrm{NCH}_{3}\right), 32.0\left(\mathrm{CH}_{3}\right.$, $\left.\mathrm{NCH}_{3}\right), 37.7\left(\mathrm{CH}_{3}, \mathrm{NCH}_{3}\right), 39.4\left(\mathrm{CH}_{3}, \mathrm{NCH}_{3}\right), 61.6\left(\mathrm{CH}_{2}\right.$, allylC), $110.9\left(\mathrm{C}, \mathrm{C}^{5}\right), 121.0\left(\mathrm{CH}\right.$, central allyl), $141.9\left(\mathrm{C}, \mathrm{C}^{4}\right), 151.4$ $(\mathrm{C}, \mathrm{C}=\mathrm{O}), 153.9(\mathrm{C}, \mathrm{C}=\mathrm{O}), 184.9$ (C, carbene).

IR (KBr): $\nu_{\mathrm{CO}}=1706,1668 \mathrm{~cm}^{-1}, \nu_{\mathrm{BF}}=1053 \mathrm{~cm}^{-1}$.

Anal. calcd for $\mathrm{C}_{21} \mathrm{H}_{29} \mathrm{BF}_{4} \mathrm{~N}_{8} \mathrm{O}_{4} \mathrm{Pd}$ : C 38.76, $\mathrm{H} 4.49, \mathrm{~N}$ 17.22. Found: C 38.50, H 4.82, N 17.35.

\section{Synthesis of complex $8 b$}

Complex 8b was prepared in an analogous manner to that described for 8a starting from $0.0157 \mathrm{~g}$ of $\left[\mathrm{Pd}(\mu-\mathrm{Cl})\left(\eta^{3} \text {-allyl }\right)\right]_{2}$, $0.0822 \mathrm{~g}$ of $3 \mathrm{~b} / \mathrm{AgBF}_{4}$ and $0.0142 \mathrm{~g}$ of KI.

$0.0599 \mathrm{~g}$ (yield $87 \%$ ) of $\mathbf{8 b}$ was obtained.

${ }^{1} \mathrm{H}-\mathrm{NMR}$ (400 MHz, $\left.T=298 \mathrm{~K}, \mathrm{CD}_{3} \mathrm{CN}, \mathrm{ppm}\right) \delta: 2.79$ (d, $2 \mathrm{H}$, $J=13.2 \mathrm{~Hz}$, anti allyl-H), $3.23\left(\mathrm{~s}, 6 \mathrm{H}, \mathrm{NCH}_{3}\right), 3.68(\mathrm{~s}, 6 \mathrm{H}$, $\mathrm{NCH}_{3}$ ), 3.97 (s, 6H, $\mathrm{NCH}_{3}$ ), 3.97 (bd, 2H, syn allyl-H), 5.39-5.61 ( $\mathrm{m}, 5 \mathrm{H}, \mathrm{NCH}_{2}$ and central allyl-H), 6.94-7.24 (m, 10H, 2Ph).

${ }^{13} \mathrm{C}\left\{{ }^{1} \mathrm{H}\right\}$-NMR $\left(T=298 \mathrm{~K}, \mathrm{CD}_{3} \mathrm{CN}, \mathrm{ppm}\right) \delta: 28.3\left(\mathrm{CH}_{3}, \mathrm{NCH}_{3}\right)$, $31.9\left(\mathrm{CH}_{3}, \mathrm{NCH}_{3}\right), 39.6\left(\mathrm{CH}_{3}, \mathrm{NCH}_{3}\right), 53.0\left(\mathrm{CH}_{2}, \mathrm{NCH}_{2}\right), 65.9$ $\left(\mathrm{CH}_{2}\right.$, allyl-C), 110.4 (C, $\left.\mathrm{C}^{5}\right), 121.2$ (CH, central allyl), 125.7-137.2 (Ph), $142.0\left(\mathrm{C}, \mathrm{C}^{4}\right), 151.2(\mathrm{C}, \mathrm{C}=\mathrm{O}), 153.2$ (C, $\mathrm{C}=\mathrm{O}), 185.3$ (C, carbene).

IR (KBr): $\nu_{\mathrm{CO}}=1709,1664 \mathrm{~cm}^{-1}, \nu_{\mathrm{BF}}=1058 \mathrm{~cm}^{-1}$.

Anal. calcd for $\mathrm{C}_{33} \mathrm{H}_{37} \mathrm{BF}_{4} \mathrm{~N}_{8} \mathrm{O}_{4} \mathrm{Pd}$ : C 49.36, $\mathrm{H}$ 4.64, N 13.96 . Found: C 49.57, H 4.12, N 14.22.

\section{Synthesis of complex 8c}

Complex 8c was prepared in an analogous manner to that described for 8a starting from $0.0157 \mathrm{~g}$ of $\left[\mathrm{Pd}(\mu-\mathrm{Cl})\left(\eta^{3} \text {-allyl }\right)\right]_{2}$, $0.0863 \mathrm{~g}$ of $3 \mathrm{c} / \mathrm{AgBF}_{4}$ and $0.0142 \mathrm{~g}$ of $\mathrm{KI}$.

$0.0592 \mathrm{~g}$ (yield $81 \%$ ) of $\mathbf{8 c}$ was obtained.

${ }^{1} \mathrm{H}-\mathrm{NMR}\left(400 \mathrm{MHz}, T=298 \mathrm{~K}, \mathrm{CD}_{3} \mathrm{CN}, \mathrm{ppm}\right) \delta: 3.02(\mathrm{~s}, 6 \mathrm{H}$, $\mathrm{NCH}_{3}$ ), $3.03(\mathrm{~d}, 2 \mathrm{H}, J=13.4 \mathrm{~Hz}$, anti allyl-H), $3.53(\mathrm{~s}, 6 \mathrm{H}$, $\left.\mathrm{NCH}_{3}\right), 4.20\left(\mathrm{~m}, 8 \mathrm{H}, \mathrm{NCH}_{3}\right.$ and syn allyl-H), 5.44 (bs, 4H, $\mathrm{NCH}_{2}$ ), 5.66 (m, 1H, central allyl-H), 7.16-7.48 (m, 10H, 2Ph). ${ }^{13} \mathrm{C}\left\{{ }^{1} \mathrm{H}\right\}-\mathrm{NMR}\left(T=298 \mathrm{~K}, \mathrm{CD}_{3} \mathrm{CN}, \mathrm{ppm}\right) \delta: 28.4\left(\mathrm{CH}_{3}, \mathrm{NCH}_{3}\right)$, $31.9\left(\mathrm{CH}_{3}, \mathrm{NCH}_{3}\right), 39.8\left(\mathrm{CH}_{3}, \mathrm{NCH}_{3}\right), 41.1\left(\mathrm{CH}_{2}, \mathrm{NCH}_{2}\right), 65.9$ 
( $\mathrm{CH}_{2}$, allyl-C), $83.8\left(\mathrm{CH}_{2}-\mathrm{C} \equiv\right), 84.7(\mathrm{C}, \mathrm{Ph}-\mathrm{C} \equiv), 110.4\left(\mathrm{C}, \mathrm{C}^{5}\right)$, 121.6 (CH, central allyl), 121.9-131.0 (Ph), $141.7\left(\mathrm{C}, \mathrm{C}^{4}\right), 150.5$ $(\mathrm{C}, \mathrm{C}=\mathrm{O}), 153.4(\mathrm{C}, \mathrm{C}=\mathrm{O}), 185.5$ (C, carbene).

IR (KBr): $\nu_{\mathrm{CO}}=1706,1667 \mathrm{~cm}^{-1}, \nu_{\mathrm{BF}}=1056 \mathrm{~cm}^{-1}$.

Anal. calcd for $\mathrm{C}_{37} \mathrm{H}_{37} \mathrm{BF}_{4} \mathrm{~N}_{8} \mathrm{O}_{4} \mathrm{Pd}$ : C 52.22, H 4.38, N 13.17. Found: C 52.37, H 4.10, N 13.55.

\section{Synthesis of complex 8d}

Complex 8d was prepared in an analogous manner to that described for 8a starting from $0.0154 \mathrm{~g}$ of $\left[\mathrm{Pd}(\mu-\mathrm{Cl})\left(\eta^{3} \text {-allyl }\right)\right]_{2}$, $0.0806 \mathrm{~g}$ of $3 \mathrm{~d} / \mathrm{AgBF}_{4}$ and $0.0139 \mathrm{~g}$ of $\mathrm{KI}$.

$0.0595 \mathrm{~g}$ (yield $88 \%$ ) of $\mathbf{8 d}$ was obtained.

${ }^{1} \mathrm{H}-\mathrm{NMR}\left(400 \mathrm{MHz}, T=298 \mathrm{~K}, \mathrm{CD}_{3} \mathrm{CN}, \mathrm{ppm}\right) \delta: 2.96(\mathrm{~d}, 2 \mathrm{H}$, $J=13.3 \mathrm{~Hz}$, anti allyl-H), $3.73\left(\mathrm{~s}, 6 \mathrm{H}, \mathrm{NCH}_{3}\right), 3.93(\mathrm{~s}, 6 \mathrm{H}$, $\left.\mathrm{NCH}_{3}\right), 4.02\left(\mathrm{~s}, 6 \mathrm{H}, \mathrm{NCH}_{3}\right), 4.13(\mathrm{~d}, 2 \mathrm{H}, J=7.4 \mathrm{~Hz}, 2$ syn allyl-H), $5.13\left(\mathrm{~s}, 4 \mathrm{H}, \mathrm{NCH}_{2}\right), 5.59(\mathrm{~m}, 1 \mathrm{H}$, central allyl-H), 7.28-7.39 (m, 10H, 2Ph).

${ }^{13} \mathrm{C}\left\{{ }^{1} \mathrm{H}\right\}-\mathrm{NMR}\left(T=298 \mathrm{~K}, \mathrm{CD}_{3} \mathrm{CN}, \mathrm{ppm}\right) \delta: 31.5\left(\mathrm{CH}_{3}, \mathrm{NCH}_{3}\right)$, $37.2\left(\mathrm{CH}_{3}, \mathrm{NCH}_{3}\right), 38.8\left(\mathrm{CH}_{3}, \mathrm{NCH}_{3}\right), 44.6\left(\mathrm{CH}_{2}, \mathrm{NCH}_{2}\right), 61.1$ $\left(\mathrm{CH}_{2}\right.$, allyl-C), 110.4 (C, $\left.\mathrm{C}^{5}\right), 120.4$ (CH, central allyl), 127.4-137.3 (Ph), 141.5 (C, $\left.\mathrm{C}^{4}\right), 150.7(\mathrm{C}, \mathrm{C}=\mathrm{O}), 153.2$ (C, $\mathrm{C}=\mathrm{O}), 184.7$ (C, carbene).

IR (KBr): $\nu_{\mathrm{CO}}=1708,1667 \mathrm{~cm}^{-1}, \nu_{\mathrm{BF}}=1058 \mathrm{~cm}^{-1}$.

Anal. calcd for $\mathrm{C}_{33} \mathrm{H}_{37} \mathrm{BF}_{4} \mathrm{~N}_{8} \mathrm{O}_{4} \mathrm{Pd}$ : $\mathrm{C}$ 49.36, $\mathrm{H}$ 4.64, N 13.96. Found: C 49.61, H 4.17, N 14.05.

\section{Growth inhibition assays}

Cell growth inhibition assays were carried out using two human ovarian cancer cell lines, A2780 and SKOV-3; A2780 cells are cisplatin-sensitive and SKOV-3 cells are cisplatinresistant. The cell lines were obtained from ATCC (Manassas, VA) and maintained in RPMI 1640 supplemented with 10\% fetal bovine serum (FBS), penicillin (100 units per mL), streptomycin $\left(100 \mu \mathrm{g} \mathrm{mL} \mathrm{mL}^{-1}\right)$ and glutamine (2 $\mathrm{mM}$ ) (complete medium); the $\mathrm{pH}$ of the medium was 7.2 and the incubation was performed at $37{ }^{\circ} \mathrm{C}$ under a $5 \% \mathrm{CO}_{2}$ atmosphere. Adherent cells were routinely used at $70 \%$ confluence and passaged every 3 days by treatment with $0.05 \%$ trypsin-EDTA (Lonza).

Pure derivatives were added at serial dilutions and incubated for 3 days. After this time, cells were washed with $1 \times$ PBS and detached with trypsin. Cells were suspended in a physiological solution and counted with a Z2 Coulter Counter (Coulter Electronics, Hialeah, FL, USA). The cell number per $\mathrm{mL}$ was determined as $\mathrm{IC}_{50}$ after 3 days of culture, when untreated cells are in the log phase of cell growth. ${ }^{23,24}$ All stock solutions were diluted in complete medium to give the final concentrations. Cisplatin was employed as a control for the cisplatin-sensitive A2780 and for the cisplatin-resistant SKOV3. Untreated cells were placed in every plate as a negative control. The cells were exposed to the compounds in $1000 \mu \mathrm{L}$ total volume for 72 hours.

\section{Apoptosis assays}

Annexin V and Dead Cell assays on A2780 and SKOV-3 cells, untreated and treated for $72 \mathrm{~h}$ with increasing doses of palladium complexes, were performed with the Muse cell analyzer
(Millipore, Billerica, MA, USA) method, according to the instructions supplied by the manufacturer. This procedure utilizes Annexin V to detect PS (PhosphatidylSerine) on the external membrane of apoptotic cells. A dead cell marker is also used as an indicator of cell membrane structural integrity. Four populations of cells can be distinguished when using this assay: live, early apoptotic, late apoptotic and dead cells. Cells were washed with sterile $1 \times$ PBS, tripsinized, resuspended in the original medium and diluted $(1: 2)$ with the one step addition of the Muse Annexin V \& Dead Cell reagent. After $20 \mathrm{~min}$ of incubation at room temperature, samples were analyzed using $0.01 \%$ Triton $\mathrm{X}$ as a positive control. ${ }^{25}$ Data from the prepared samples were acquired and recorded utilizing the Annexin V and Dead Cell Software Module (Millipore, Billerica, MA, USA).

\section{Crystal structure determination}

The crystal data of $\mathbf{4 a}$ and $\mathbf{8 d}$ were collected at $100 \mathrm{~K}$ at the XRD1 beamline of the Elettra Synchrotron, Trieste (Italy). ${ }^{26}$ The data sets were integrated and corrected for Lorentz and polarization effects using the XDS package. ${ }^{27}$ Data have been scaled using the CCP4 Aimless code. ${ }^{28}$ Crystals of $8 \mathbf{d}$ showed significant radiation damage upon exposure to X-rays; therefore data from three different crystals were merged to obtain a complete set of data. The structures were solved by direct methods using the SHELXT program ${ }^{29}$ and refined using fullmatrix least-squares with all non-hydrogen atoms anisotropically and hydrogens included on calculated positions, riding on their carrier atoms. Geometric restraints on bond lengths and angles (DFIX, DANG) have been used in a 4 a model for disordered fragments (i.e. $\mathrm{CH}_{2} \mathrm{Cl}_{2}$ solvent molecule and $\mathrm{BF}_{4}{ }^{-}$ ion). Thermal parameters restraints (SIMU, ISOR and isotropic treatment of disordered allyl fragments) have been introduced to successfully refine the structure of $\mathbf{8 d}$, impaired by electron density noise due to radiation damage effects and non-merohedral twinning (the structure was refined as a 2-component twin, with domains related by twofold axis $\left[\begin{array}{lll}1 & 0 & 0\end{array}\right]$ and twin fraction refined to $7 \%$ ). All calculations were performed using SHELXL-2017/1. ${ }^{30}$ The Coot program was used for structure building. ${ }^{31}$ The crystal data are given in Table $\mathrm{S} 1 . \dagger$ Pictures were prepared using the Ortep $3^{32}$ software.

Crystallographic data have been deposited at the Cambridge Crystallographic Data Centre and allocated the deposition numbers CCDC 1825947 (for 4a) and 1825948 (for $\mathbf{8 d})+\dagger$

\section{Conflicts of interest}

There are no conflicts to declare.

\section{References}

1 (a) B. Rosenberg, L. van Camp and T. Krigas, Nature, 1965, 205, 698; (b) B. Rosenberg, in Cisplatin, Chemistry and 
Biochemistry of a Leading Anticancer Drug, ed. S. J. Lippert, Wiley-VCH, Weinheim, 1999.

2 (a) Z. J. Guo and P. J. Sadler, Adv. Inorg. Chem., 2000, 49, 183; (b) X. Wang and Z. Guo, in Bioinorganic Medicinal Chemistry, ed. E. Alessio, Wiley-VCH, Weinheim, 2008, ch. 4; (c) I. Bratsos, T. Gianferrara, E. Alessio, C. G. Hartinger, M. A. Jakupec and B. K. Keppler, in Bioinorganic Medicinal Chemistry, ed. E. Alessio, Wiley-VCH Verlag $\mathrm{GmbH} \&$ Co. KGaA, Weinheim, 2008, ch. 5;; (d) M. A. Jakupec, M. Galanski, V. B. Arion, C. G. Hartinger and B. K. Keppler, Dalton Trans., 2008, 183; (e) F. Arnesano and G. Natile, Pure Appl. Chem., 2008, 80, 2715; (f) J. C. Dabrowiak, Metals in Medicine, John Wiley \& Sons, Ltd, Chichester, 2009; $(g)$ T. Gianferrara, I. Bratsos and E. Alessio, Dalton Trans., 2009, 7588; (h) A. Casini, C. G. Hartinger, A. A. Nazarov and P. J. Dyson, Top. Organomet. Chem., 2010, 32, 57; (i) P. Zhang and P. J. Sadler, J. Organomet. Chem., 2017, 839, 5 .

3 (a) M. A. Fuentes, C. Alonso and J. M. Perez, Chem. Rev., 2003, 103, 645; (b) Y. Jung and S. J. Lippard, Chem. Rev., 2007, 107, 1387; (c) J. Reedijk, Eur. J. Inorg. Chem., 2009, 1303.

4 P. J. O'Dwyer, J. P. Stevenson and S. W. Johnson, in Cisplatin: Chemistry and Biochemistry of a Leading Anticancer Drug, ed. B. Lippert, Wiley-VCH, Weinheim, 1999.

5 (a) P. J. Dyson and G. Sava, Dalton Trans., 2006, 1929; (b) A. Bergamo and G. Sava, Dalton Trans., 2007, 1267.

6 (a) S. Ray, R. Mohan, J. K. Singh, M. K. Samantaray, M. M. Shaikh, D. Panda and P. Ghosh, J. Am. Chem. Soc., 2007, 129, 15042; (b) N. Cutillas, G. S. Yellol, C. de Haro, C. Vicente, V. Rodríguez and J. Ruiz, Coord. Chem. Rev., 2013, 257, 2784; (c) A. R. Kapdi and I. J. S. Fairlamb, Chem. Soc. Rev., 2014, 43, 4751; (d) S. Medici, M. Peana, V. M. Nurchi, J. I. Lachowicz, G. Crisponi and M. A. Zoroddua, Coord. Chem. Rev., 2015, 284, 329; (e) T. Fong, C. Lok, C. Y. Chung, Y. E. Fung, P. Chow, P. Wan and C. Che, Angew. Chem., Int. Ed., 2016, 55, 11935.

7 (a) H. Hohamann and R. van Eldik, Inorg. Chim. Acta, 1990, 174, 87; (b) H. Hohamann, H. Suvachittanont and R. van Eldik, Inorg. Chim. Acta, 1990, 177, 5.

8 (a) W. A. Hermann, Angew. Chem., Int. Ed., 2002, 41, 1290; (b) L. Cavallo, A. Correa, C. Costabile and H. Jacobsen, J. Organomet. Chem., 2005, 690, 5407; (c) S. Díez-González and S. P. Nolan, Coord. Chem. Rev., 2007, 251, 874; (d) P. de Frémont, N. Marion and S. P. Nolan, Coord. Chem. Rev., 2009, 253, 862.

9 (a) J. G. Cropp, Am. J. Med., 1996, 100(1A), 195; (b) M. Teyssot, A. Jarrousse, M. Manin, A. Chevry, S. Roche, F. Norre, C. Beaudoin, L. Morel, D. Boyer, R. Mahioue and A. Gautier, Dalton Trans., 2009, 6894; (c) A. John and P. Ghosh, Dalton Trans., 2010, 39, 7183; (d) S. B. Aher, P. N. Muskawar, K. Thenmozhi and P. R. Bhagat, Eur. J. Med. Chem., 2014, 81, 408.

10 (a) A. Kascatan-Nebioglu, M. J. Panzner, J. C. Garrison, C. A. Tessier and W. J. Youngs, Organometallics, 2004, 23, 1928; (b) J. Schütz and W. A. Herrmann, J. Organomet.
Chem., 2004, 689, 2995; (c) A. Kascatan-Nebioglu, A. Melaiye, K. Hindi, S. Durmus, M. J. Panzner, L. A. Hogue, R. J. Mallett, C. E. Hovis, M. Coughenour, S. D. Crosby, A. Milsted, D. L. Ely, C. A. Tessier, C. L. Cannon and W. J. Youngs, J. Med. Chem., 2006, 49, 6811; (d) F.-T. Luo and H.-K. Lo, J. Organomet. Chem., 2011, 696, 1262; (e) A. Makhloufi, W. Frank and C. Ganter, Organometallics, 2012, 31, 7272; $(f)$ A. Monney and M. Albrecht, Coord. Chem. Rev., 2013, 257, 2420; $(g)$ S. E. Flowers and B. M. Cossairt, Organometallics, 2014, 33, 4341; (h) J. Zhang, J. K. Muenzner, M. A. Abu el Maaty, B. Karge, R. Schobert, S. Wölf and I. Ott, Dalton Trans., 2016, 45, 13161; ( $i$ ) E. Mohammadi and B. Movassagh, J. Organomet. Chem., 2016, 822, 62; (j) A. Szadkowska, S. Staszko, E. Zaorska and R. Pawlowski, RSC Adv., 2016, 6, 44248.

11 (a) T. A. K. Al-Allaf and L. J. Rashan, Eur. J. Med. Chem., 1998, 33, 817; (b) A. Valentini, F. Conforti, A. Crispini, A. De Martino, R. Condello, C. Stellitano, G. Rotillo, M. Ghedini, G. Federici, S. Bernardini and D. Pucci, J. Med. Chem., 2009, 52, 484; (c) A. Monney and M. Albrecht, Coord. Chem. Rev., 2013, 257, 2420; (d) M. Tanaka, H. Kataoka, S. Yano, H. Ohi, K. Kawamoto, T. Shibahara, T. Mizoshita, Y. Mori, S. Tanida, T. Kamiya and T. Joh, BMC Cancer, 2013, 13, 327.

12 (a) D. A. Krogstad, J. Cho, A. J. DeBoer, J. A. Klitzke, W. R. Sanow, H. A. Williams and J. A. Halfen, Inorg. Chim. Acta, 2006, 359, 136; (b) E. Vergara, S. Miranda, F. Mohr, E. Cerrada, E. R. T. Tiekink, P. Romero, A. Mendía and M. Laguna, Eur. J. Inorg. Chem., 2007, 2926; (c) J. Spencer, A. Casini, O. Zava, R. P. Rathnam, S. K. Velhanda, M. Pfeffer, S. K. Callear, M. B. Hursthouseand and P. Dyson, Dalton Trans., 2009, 10731; (d) J. Lasri, M. J. Fernández Rodríguez, M. F. C. Guedes da Silva, P. Smolenski, M. N. Kopylovich, J. J. R. Fraústo da Silva and A. J. L. Pombeiro, J. Organomet. Chem., 2011, 696, 3513; (e) M. Carreira, R. Calvo-Sanjuan, M. Sanauí, I. Marzo and M. Contel, Organometallics, 2012, 31, 5772; $(f)$ E. Guerrero, S. Miranda, S. Luüttenberg, N. Froöhlich, J. Koenen, F. Mohr, E. Cerrada, M. Laguna and A. Mendía, Inorg. Chem., 2013, 52, 6635; (g) J. Braddock-Wilking, S. Acharya and N. P. Rath, Polyhedron, 2014, 79, 16; (h) V. Ferretti, M. Fogagnolo, A. Marchi, L. Marvelli, F. Sforza and P. Bergamini, Inorg. Chem., 2014, 53(10), 4881.

$13 \mathrm{~J}$. Tsuji, in Handbook of Organopalladium Chemistry for Organic Synthesis, ed. E. Negishi, Wiley-Interscience, New York, 2002, p. 1669.

14 (a) B. Crociani, S. Antonaroli, F. Di Bianca, L. Canovese, F. Visentin and P. Uguagliati, J. Chem. Soc., Dalton Trans., 1994, 1145; (b) L. Canovese, F. Visentin, P. Uguagliati, G. Chessa and A. Pesce, J. Organomet. Chem., 1998, 566, 61; (c) L. Canovese, G. Chessa, C. Santo, F. Visentin and P. Uguagliati, Organometallics, 2002, 21, 4342; (d) F. Visentin and A. Togni, Organometallics, 2007, 26, 3746; (e) L. Canovese, F. Visentin, C. Santo, G. Chessa and V. Bertolasi, Organometallics, 2010, 29, 3027; L. Canovese, 
F. Visentin, C. Levi and A. Dolmella, Dalton Trans., 2011, 40, 966; $(f)$ L. Canovese, F. Visentin, C. Levi, C. Santo and V. Bertolasi, J. Organomet. Chem., 2013, 732, 27; (g) L. Canovese, F. Visentin, C. Santo and V. Bertolasi, Organometallics, 2014, 33, 1700; $(h)$ L. Canovese, F. Visentin, T. Scattolin, C. Santo and V. Bertolasi, Polyhedron, 2016, 119, 377.

15 (a) Y. G. Yuan, Q. L. Peng and S. Gurunathan, Int. J. Nanomed., 2017, 12, 6503; (b) O. Kacar, B. Cevatemre, I. Hatipoglu, N. Arda, E. Ulukaya, V. T. Yilmaz and C. Acilan, Bioorg. Med. Chem., 2017, 25, 1770; (c) Y. G. Sun, Y. N. Sun, L. X. You, Y. N. Liu, F. Ding, B. Y. Ren, G. Xiong, V. Dragutan and I. Dragutan, J. Inorg. Biochem., 2016, 164, 129.

16 (a) B. Bertrand, L. Stefan, M. Pirrotta, D. Monchaud, E. Bodio, P. Richard, P. Le Gendre, E. Warmerdam, M. H. de Jager, G. M. M. Groothuis, M. Picquet and A. Casini, Inorg. Chem., 2014, 53, 2296; (b) J. Reisch, Arzneimittelforschung, 1968, 18, 1485.

17 T. Fujii, T. Saito and K. Tamura, Chem. Pharm. Bull., 1991, 39, 2855.

18 J. Schütz and W. A. Herrmann, J. Organomet. Chem., 2004, 689, 2995.

19 T. Scattolin, L. Canovese, F. Visentin, S. Paganelli, P. Canton and N. Demitri, Appl. Organomet. Chem., 2018, 32, e4034.

20 B. Crociani, S. Antonaroli, G. Bandoli, L. Canovese, F. Visentin and P. Uguagliati, Organometallics, 1999, 18, E 44-34.

21 A. T. Normand, A. Stasch, L. Ooi and K. J. Cavell, Organometallics, 2008, 27, 6507.

22 A. Spek, Acta Crystallogr., Sect. D: Biol. Crystallogr., 2009, 65, 148.

23 M. T. Khan, M. Borgatti, N. Bianchi and R. Gambari, Evid. Based Complement. Alternat. Med., 2008, 5, 303.

24 P. Bergamini, L. Marvelli, V. Ferretti, C. Gemmo, R. Gambari, Y. Hushcha and I. Lampronti, Dalton Trans., 2016, 45, 10752.

25 R. Milani, A. Marcellini, G. Montagner, A. Baldisserotto, S. Manfredini, R. Gambari and I. Lampronti, Eur. J. Pharm. Sci., 2015, 78, 225.

26 A. Lausi, M. Polentarutti, S. Onesti, J. R. Plaisier, E. Busetto, G. Bais, L. Barba, A. Cassetta, G. Campi, D. Lamba, A. Pifferi, S. C. Mande, D. D. Sarma, S. M. Sharma and G. Paolucci, Eur. Phys. J. Plus, 2015, 130, 1.
27 W. Kabsch, Acta Crystallogr., Sect. D: Biol. Crystallogr., 2010, 66, 125.

28 (a) M. D. Winn, C. C. Ballard, K. D. Cowtan, E. J. Dodson, P. Emsley, P. R. Evans, R. M. Keegan, E. B. Krissinel, A. G. W. Leslie, A. McCoy, S. J. McNicholas, G. N. Murshudov, N. S. Pannu, E. A. Potterton, H. R. Powell, R. J. Read, A. Vagin and K. S. Wilson, Acta Crystallogr., Sect. D: Biol. Crystallogr., 2011, 67, 235; (b) P. R. Evans and G. N. Murshudov, Acta Crystallogr., Sect. D: Biol. Crystallogr., 2013, 69, 1204.

29 G. M. Sheldrick, Acta Crystallogr., Sect. A: Found. Adv., 2015, $71,3$.

30 G. M. Sheldrick, Acta Crystallogr., Sect. C: Struct. Chem., 2015, 71, 3.

31 P. Emsley, B. Lohkamp, W. Scott and K. Cowtan, Acta Crystallogr., Sect. D: Biol. Crystallogr., 2010, 66, 486.

32 L. Farrugia, J. Appl. Crystallogr., 2012, 45, 849.

33 R. Milani, E. Brognara, E. Fabbri, A. Finotti, M. Borgatti, I. Lampronti, G. Marzaro, A. Chilin, K. K. Lee, S. H. Kok, C. H. Chui and R. Gambari, Oncol. Res., 2017, DOI: 10.3727/096504017X14928634401187.

34 A. Bertucci, E. A. Prasetyanto, D. Septiadi, A. Manicardi, E. Brognara, R. Gambari, R. Corradini and L. De Cola, Small, 2015, 11, 5687.

35 E. Brognara, E. Fabbri, E. Bazzoli, G. Montagner, C. Ghimenton, A. Eccher, C. Cantù, A. Manicardi, N. Bianchi, A. Finotti, G. Breveglieri, M. Borgatti, R. Corradini, V. Bezzerri, G. Cabrini and R. Gambari, J. Neurooncol., 2014, 118, 19.

36 X. F. Zhang, Q. Yan, W. Shen and S. Gurunathan, Int. J. Mol. Sci., 2016, $17(8), 1354$.

37 C. Mundhenke, M. T. Weigel, K. H. Sturner, F. Roesel, I. Meinhold-Heerlein, D. O. Bauerschlag, C. Schem, F. Hilpert, W. Jonat and N. Maass, J. Cancer Res. Clin. Oncol., 2008, 134, 1397.

38 M. Cuello, S. A. Ettenberg, M. M. Nau and S. Lipkowitz, Gynecol. Oncol., 2001, 81, 380.

39 Y. H. Ling, Y. Yang, C. Tornos, B. Singh and R. Perez-Soler, Cancer Res., 1998, 58, 3633.

40 Y. G. Yuan, Q. L. Peng and S. Gurunathan, Int. J. Nanomed., 2017, 12, 6502.

41 M. Sui, X. Xiong, A. S. Kraft and W. Fan, Cancer Biol. Ther., 2006, 5, 1015. 